\title{
A Class of Modified Ratio Estimators for Estimation of Population Variance
}

\author{
J. SUBRAMANI AND G. KUMARAPANDIYAN
}

\begin{abstract}
In this paper we have proposed a class of modified ratio type variance estimators for estimation of population variance of the study variable using the known parameters of the auxiliary variable. The bias and mean squared error of the proposed estimators are obtained and also derived the conditions for which the proposed estimators perform better than the traditional ratio type variance estimator and existing modified ratio type variance estimators. Further we have compared the proposed estimators with that of the traditional ratio type variance estimator and existing modified ratio type variance estimators for certain natural populations.
\end{abstract}

Mathematics Subject Classification 2000: 62 D05

General Terms: Auxiliary variable, Bias, Mean squared error

Additional Key Words and Phrases: Coefficient of variation, Kurtosis, Median, Natural populations, Simple random sampling, Skewness

\section{INTRODUCTION}

It is common practice to use the auxiliary variable for improving the precision of the estimate of a parameter. When the information on an auxiliary variable $\mathrm{X}$ is known, a number of estimators such as ratio, product and linear regression estimators are available in the literature. When the correlation between the study variable and the auxiliary variable is positive, ratio method of estimation is quite effective. Consider a finite population $U=\left\{U_{1}, U_{2}, \ldots, U_{N}\right\}$ of $N$ distinct and identifiable units. Let $Y$ be a real variable with value $Y_{i}$ measured on $U_{i}, i=1,2,3, \ldots, N$ giving a vector $Y=$ $\left\{Y_{1}, Y_{2}, \ldots, Y_{N}\right\}$. The problem is to estimate the population mean $\bar{Y}=\frac{1}{N} \sum_{i=1}^{N} Y_{i}$ on the basis of a random sample selected from the population $U$ or its variance $S_{y}^{2}=$ $\frac{1}{(\mathrm{~N}-1)} \sum_{\mathrm{i}=1}^{\mathrm{N}}\left(\mathrm{Y}_{\mathrm{i}}-\overline{\mathrm{Y}}\right)^{2}$. When there is no additional information on the auxiliary variable available, the simplest estimator of population mean or variance is the simple random sample mean or variance without replacement. As stated earlier, if an auxiliary variable $\mathrm{X}$ closely related to the study variable $\mathrm{Y}$ is available and $\mathrm{X}$ is easy

${ }^{1}$ This research was supported by the University Grants Commission, New Delhi through Major Research Project. 
to obtain then one can use ratio, product and regression estimators to improve the performance of the estimator of the study variable. Estimating the finite population variance has great significance in various fields such as Industry, Agriculture, Medical and Biological sciences. In this paper, we consider the problem of estimation of the population variance and use the auxiliary information to improve the efficiency of the estimator of population variance.

Before discussing further about the traditional ratio type variance estimator, existing modified ratio type variance estimators and the proposed modified ratio type variance estimators, the notations to be used in this paper are described below:

- $\mathrm{N}$ - Population size

- $\mathrm{n}$ - Sample size

- $\gamma=\frac{(1-\mathrm{f})}{\mathrm{n}}$

- $\mathrm{Y}$ - Study variable

- $X-$ Auxiliary variable

- $\overline{\mathrm{X}}, \overline{\mathrm{Y}}-$ Population means

- $\overline{\mathrm{X}}, \overline{\mathrm{y}}-$ Sample means

- $S_{\mathrm{y}}^{2}, S_{\mathrm{x}}^{2}-$ Population variances

- $\mathrm{s}_{\mathrm{y}}^{2}, \mathrm{~s}_{\mathrm{x}}^{2}-$ Sample variances

- $\mathrm{C}_{\mathrm{X}}, \mathrm{C}_{\mathrm{y}}-$ Coefficient of variations

- $\lambda_{\mathrm{rs}}=\frac{\mu_{\mathrm{rs}}}{\mu_{20}^{\mathrm{r} / 2} \mu_{02}^{s / 2}}$

- $\mu_{\mathrm{rs}}=\frac{1}{\mathrm{~N}} \sum_{\mathrm{i}=1}^{\mathrm{N}}\left(\mathrm{y}_{\mathrm{i}}-\overline{\mathrm{Y}}\right)^{\mathrm{r}}\left(\mathrm{x}_{\mathrm{i}}-\overline{\mathrm{X}}\right)^{\mathrm{s}}$

- $\beta_{1(\mathrm{x})}=\frac{\mu_{03}^{2}}{\mu_{02}^{3}}$, Skewness of the auxiliary variable

- $\mathrm{M}_{\mathrm{d}}-$ Median of the auxiliary variable

- $\mathrm{Q}_{1}$-First (lower) quartile of the auxiliary variable

- $\mathrm{Q}_{3}$-Third (upper) quartile of the auxiliary variable

- $\mathrm{Q}_{\mathrm{r}}$-Inter-quartile range of the auxiliary variable

- $Q_{d}-$ Semi-quartile range of the auxiliary variable

- $\mathrm{Q}_{\mathrm{a}}-$ Semi-quartile average of the auxiliary variable

- $\mathrm{D}_{\mathrm{i}}-\mathrm{i}^{\text {th }}$ Decile of the auxiliary variable

- $\mathrm{B}()-$. Bias of the estimator

- MSE(.) - Mean squared error of the estimator

- $\widehat{S}_{\mathrm{R}}^{2}-$ Traditional ratio type variance estimator of $\mathrm{S}_{\mathrm{y}}^{2}$

- $\widehat{S}_{\mathrm{i}}^{2}-$ Existing modified ratio type variance estimator of $\mathrm{S}_{\mathrm{y}}^{2}$ 
- $\quad \widehat{\mathrm{S}}_{\mathrm{p}_{\mathrm{i}}}^{2}-$ Proposed modified ratio type variance estimator of $\mathrm{S}_{\mathrm{y}}^{2}$

Isaki (1983) suggested a ratio type variance estimator for the population variance $\mathrm{S}_{\mathrm{y}}^{2}$ when the population variance $S_{x}^{2}$ of the auxiliary variable $X$ is known together with its bias and mean squared error are given below:

$\widehat{S}_{\mathrm{R}}^{2}=\mathrm{s}_{\mathrm{y}}^{2} \frac{\mathrm{S}_{\mathrm{x}}^{2}}{\mathrm{~s}_{\mathrm{x}}^{2}}$

$\mathrm{B}\left(\widehat{\mathrm{S}}_{\mathrm{R}}^{2}\right)=\gamma \mathrm{S}_{\mathrm{y}}^{2}\left[\left(\beta_{2(\mathrm{x})}-1\right)-\left(\lambda_{22}-1\right)\right]$

$\operatorname{MSE}\left(\widehat{\mathrm{S}}_{\mathrm{R}}^{2}\right)=\gamma \mathrm{S}_{\mathrm{y}}^{4}\left[\left(\beta_{2(\mathrm{y})}-1\right)+\left(\beta_{2(\mathrm{x})}-1\right)-2\left(\lambda_{22}-1\right)\right]$

where $\beta_{2(\mathrm{y})}=\frac{\mu_{40}}{\mu_{20}^{2}}, \beta_{2(\mathrm{x})}=\frac{\mu_{04}}{\mu_{02}^{2}}, \lambda_{22}=\frac{\mu_{22}}{\mu_{20} \mu_{02}}$

The ratio type variance estimator given in (1.1) is used to improve the precision of the estimate of the population variance compared to simple random sampling when there exists a positive correlation between $\mathrm{X}$ and $\mathrm{Y}$. Further improvements are also achieved on the ratio estimator by introducing a number of modified ratio estimators with the use of known parameters like Coefficient of Variation, Coefficient of Kurtosis, Median, Quartiles and Deciles. The problem of constructing efficient estimators for the population variance has been widely discussed by various authors such as Agarwal and Sithapit (1995), Ahmed et al. (2000), Al-Jararha and Al-Haj Ebrahem (2012), Arcos et al (2005), Cochran (1977), Das and Tripathi (1978), Garcia and Cebrain (1997), Gupta and Shabbir (2008), Isaki (1983), Kadilar and Cingi (2006a,b), Murthy (1967), Prasad and Singh (1990), Reddy (1974), Shabbir and Gupta (2006), Singh and Solanki (2013), Singh and Chaudhary (1986), Singh et al. (1988), Subramani and Kumarapandiyan (2012a,b,c,2013), tailor and Shrama (2012), Upadhyaya and Singh (1999, 2001, 2006), Wolter (1985) and Yadav and Kadilar (2013a,b).

The following table contains all modified ratio type variance estimators using known population parameters of the auxiliary variable in which some of the estimators are already suggested in the literature, remaining estimators are introduced in this paper 
Table 1: Modified ratio type estimators for estimating population variance with the bias and mean squared error

\begin{tabular}{|c|c|c|}
\hline Estimator & Bias - B(.) & Mean squared error MSE(.) \\
\hline $\begin{array}{l}\hat{S}_{1}^{2}=s_{y}^{2}\left[\frac{S_{x}^{2}+C_{x}}{s_{x}^{2}+C_{x}}\right] \\
\text { Kadilar and Cingi } \\
(2006 b)\end{array}$ & $\gamma S_{y}^{2} \delta_{1}\left[\delta_{1}\left(\beta_{2(\mathrm{x})}-1\right)-\left(\lambda_{22}-1\right)\right]$ & $\gamma S_{\mathrm{y}}^{4}\left[\left(\beta_{2(\mathrm{y})}-1\right)+\delta_{1}^{2}\left(\beta_{2(\mathrm{x})}-1\right)-2 \delta_{1}\left(\lambda_{22}-1\right)\right]$ \\
\hline $\begin{array}{l}\widehat{S}_{2}^{2}=s_{y}^{2}\left[\frac{S_{x}^{2}+\beta_{2(x)}}{s_{x}^{2}+\beta_{2(x)}}\right] \\
\text { Upadhyaya and Singh } \\
\text { (1999) }\end{array}$ & $\gamma S_{y}^{2} \delta_{2}\left[\delta_{2}\left(\beta_{2(\mathrm{x})}-1\right)-\left(\lambda_{22}-1\right)\right]$ & $\gamma S_{\mathrm{y}}^{4}\left[\left(\beta_{2(\mathrm{y})}-1\right)+\delta_{2}^{2}\left(\beta_{2(\mathrm{x})}-1\right)-2 \delta_{2}\left(\lambda_{22}-1\right)\right]$ \\
\hline$\widehat{S}_{3}^{2}=s_{y}^{2}\left[\frac{S_{x}^{2}+\beta_{1(x)}}{S_{x}^{2}+\beta_{1(x)}}\right]$ & $\gamma S_{y}^{2} \delta_{3}\left[\delta_{3}\left(\beta_{2(\mathrm{x})}-1\right)-\left(\lambda_{22}-1\right)\right]$ & $\gamma S_{y}^{4}\left[\left(\beta_{2(y)}-1\right)+\delta_{3}^{2}\left(\beta_{2(x)}-1\right)-2 \delta_{3}\left(\lambda_{22}-1\right)\right]$ \\
\hline$\widehat{S}_{4}^{2}=s_{y}^{2}\left[\frac{S_{x}^{2}+\rho}{s_{x}^{2}+\rho}\right]$ & $\gamma S_{y}^{2} \delta_{4}\left[\delta_{4}\left(\beta_{2(\mathrm{x})}-1\right)-\left(\lambda_{22}-1\right)\right]$ & $\gamma S_{y}^{4}\left[\left(\beta_{2(y)}-1\right)+\delta_{4}^{2}\left(\beta_{2(x)}-1\right)-2 \delta_{4}\left(\lambda_{22}-1\right)\right]$ \\
\hline$\hat{S}_{5}^{2}=s_{y}^{2}\left[\frac{S_{x}^{2}+S_{x}}{s_{x}^{2}+S_{x}}\right]$ & $\gamma S_{y}^{2} \delta_{5}\left[\delta_{5}\left(\beta_{2(\mathrm{x})}-1\right)-\left(\lambda_{22}-1\right)\right]$ & $\gamma S_{y}^{4}\left[\left(\beta_{2(y)}-1\right)+\delta_{5}^{2}\left(\beta_{2(x)}-1\right)-2 \delta_{5}\left(\lambda_{22}-1\right)\right]$ \\
\hline $\begin{array}{l}\hat{S}_{6}^{2}=s_{y}^{2}\left[\frac{S_{x}^{2}+M_{d}}{s_{x}^{2}+M_{d}}\right] \\
\text { Subramani and } \\
\text { Kumarapandiyan } \\
\text { (2012a) }\end{array}$ & $\gamma S_{y}^{2} \delta_{6}\left[\delta_{6}\left(\beta_{2(\mathrm{x})}-1\right)-\left(\lambda_{22}-1\right)\right]$ & $\gamma S_{\mathrm{y}}^{4}\left[\left(\beta_{2(\mathrm{y})}-1\right)+\delta_{6}^{2}\left(\beta_{2(\mathrm{x})}-1\right)-2 \delta_{6}\left(\lambda_{22}-1\right)\right]$ \\
\hline $\begin{array}{l}\hat{S}_{7}^{2}=s_{y}^{2}\left[\frac{S_{x}^{2}+Q_{1}}{S_{x}^{2}+Q_{1}}\right] \\
\text { Subramani and } \\
\text { Kumarapandiyan } \\
\text { (2012b) }\end{array}$ & $\gamma S_{y}^{2} \delta_{7}\left[\delta_{7}\left(\beta_{2(\mathrm{x})}-1\right)-\left(\lambda_{22}-1\right)\right]$ & $\gamma S_{\mathrm{y}}^{4}\left[\left(\beta_{2(\mathrm{y})}-1\right)+\delta_{7}^{2}\left(\beta_{2(\mathrm{x})}-1\right)-2 \delta_{7}\left(\lambda_{22}-1\right)\right]$ \\
\hline
\end{tabular}




\begin{tabular}{|c|c|c|}
\hline $\begin{array}{l}\hat{S}_{8}^{2}=s_{y}^{2}\left[\frac{S_{x}^{2}+Q_{3}}{s_{x}^{2}+Q_{3}}\right] \\
\text { Subramani and } \\
\text { Kumarapandiyan } \\
\text { (2012b) }\end{array}$ & $\gamma S_{y}^{2} \delta_{8}\left[\delta_{8}\left(\beta_{2(x)}-1\right)-\left(\lambda_{22}-1\right)\right]$ & $\gamma S_{\mathrm{y}}^{4}\left[\left(\beta_{2(\mathrm{y})}-1\right)+\delta_{8}^{2}\left(\beta_{2(\mathrm{x})}-1\right)-2 \delta_{8}\left(\lambda_{22}-1\right)\right]$ \\
\hline $\begin{array}{l}\hat{S}_{9}^{2}=s_{y}^{2}\left[\frac{S_{x}^{2}+Q_{r}}{s_{x}^{2}+Q_{r}}\right] \\
\text { Subramani and } \\
\text { Kumarapandiyan } \\
\text { (2012b) }\end{array}$ & $\gamma S_{y}^{2} \delta_{9}\left[\delta_{9}\left(\beta_{2(\mathrm{x})}-1\right)-\left(\lambda_{22}-1\right)\right]$ & $\gamma S_{\mathrm{y}}^{4}\left[\left(\beta_{2(\mathrm{y})}-1\right)+\delta_{9}^{2}\left(\beta_{2(\mathrm{x})}-1\right)-2 \delta_{9}\left(\lambda_{22}-1\right)\right]$ \\
\hline $\begin{array}{l}\widehat{S}_{10}^{2}=s_{y}^{2}\left[\frac{S_{x}^{2}+Q_{d}}{S_{x}^{2}+Q_{d}}\right] \\
\text { Subramani and } \\
\text { Kumarapandiyan } \\
\text { (2012b) }\end{array}$ & $\gamma S_{y}^{2} \delta_{10}\left[\delta_{10}\left(\beta_{2(\mathrm{x})}-1\right)-\left(\lambda_{22}-1\right)\right]$ & $\gamma S_{\mathrm{y}}^{4}\left[\left(\beta_{2(\mathrm{y})}-1\right)+\delta_{10}^{2}\left(\beta_{2(\mathrm{x})}-1\right)-2 \delta_{10}\left(\lambda_{22}-1\right)\right]$ \\
\hline $\begin{array}{l}\widehat{S}_{11}^{2}=s_{y}^{2}\left[\frac{S_{x}^{2}+Q_{a}}{S_{x}^{2}+Q_{a}}\right] \\
\text { Subramani and } \\
\text { Kumarapandiyan } \\
\text { (2012b) }\end{array}$ & $\gamma S_{y}^{2} \delta_{11}\left[\delta_{11}\left(\beta_{2(x)}-1\right)-\left(\lambda_{22}-1\right)\right]$ & $\gamma S_{y}^{4}\left[\left(\beta_{2(y)}-1\right)+\delta_{11}^{2}\left(\beta_{2(x)}-1\right)-2 \delta_{11}\left(\lambda_{22}-1\right)\right]$ \\
\hline $\begin{array}{l}\hat{S}_{12}^{2}=s_{y}^{2}\left[\frac{S_{x}^{2}+D_{1}}{S_{x}^{2}+D_{1}}\right] \\
\text { Subramani and } \\
\text { Kumarapandiyan } \\
\text { (2012c) }\end{array}$ & $\gamma S_{\mathrm{y}}^{2} \delta_{12}\left[\delta_{12}\left(\beta_{2(\mathrm{x})}-1\right)-\left(\lambda_{22}-1\right)\right]$ & $\gamma S_{\mathrm{y}}^{4}\left[\left(\beta_{2(\mathrm{y})}-1\right)+\delta_{12}^{2}\left(\beta_{2(\mathrm{x})}-1\right)-2 \delta_{12}\left(\lambda_{22}-1\right)\right]$ \\
\hline $\begin{array}{l}\hat{\mathrm{S}}_{13}^{2}=\mathrm{s}_{\mathrm{y}}^{2}\left[\frac{\mathrm{S}_{\mathrm{x}}^{2}+\mathrm{D}_{2}}{\mathrm{~S}_{\mathrm{x}}^{2}+\mathrm{D}_{2}}\right] \\
\text { Subramani and } \\
\text { Kumarapandiyan } \\
(2012 \mathrm{c})\end{array}$ & $\gamma S_{\mathrm{y}}^{2} \delta_{13}\left[\delta_{13}\left(\beta_{2(\mathrm{x})}-1\right)-\left(\lambda_{22}-1\right)\right]$ & $\gamma S_{\mathrm{y}}^{4}\left[\left(\beta_{2(\mathrm{y})}-1\right)+\delta_{13}^{2}\left(\beta_{2(\mathrm{x})}-1\right)-2 \delta_{13}\left(\lambda_{22}-1\right)\right]$ \\
\hline $\begin{array}{l}\hat{S}_{14}^{2}=s_{y}^{2}\left[\frac{S_{x}^{2}+D_{3}}{S_{x}^{2}+D_{3}}\right] \\
\text { Subramani and } \\
\text { Kumarapandiyan } \\
\text { (2012c) }\end{array}$ & $\gamma S_{\mathrm{y}}^{2} \delta_{14}\left[\delta_{14}\left(\beta_{2(\mathrm{x})}-1\right)-\left(\lambda_{22}-1\right)\right]$ & $\gamma S_{y}^{4}\left[\left(\beta_{2(y)}-1\right)+\delta_{14}^{2}\left(\beta_{2(\mathrm{x})}-1\right)-2 \delta_{14}\left(\lambda_{22}-1\right)\right]$ \\
\hline $\begin{array}{l}\hat{S}_{15}^{2}=s_{y}^{2}\left[\frac{S_{x}^{2}+D_{4}}{S_{x}^{2}+D_{4}}\right] \\
\text { Subramani and } \\
\text { Kumarapandiyan } \\
\text { (2012c) }\end{array}$ & $\gamma S_{y}^{2} \delta_{15}\left[\delta_{15}\left(\beta_{2(\mathrm{x})}-1\right)-\left(\lambda_{22}-1\right)\right]$ & $\gamma S_{y}^{4}\left[\left(\beta_{2(y)}-1\right)+\delta_{15}^{2}\left(\beta_{2(x)}-1\right)-2 \delta_{15}\left(\lambda_{22}-1\right)\right]$ \\
\hline $\begin{array}{l}\hat{S}_{16}^{2}=s_{y}^{2}\left[\frac{S_{x}^{2}+D_{5}}{S_{x}^{2}+D_{5}}\right] \\
\text { Subramani and } \\
\text { Kumarapandiyan } \\
\text { (2012c) }\end{array}$ & $\gamma S_{\mathrm{y}}^{2} \delta_{16}\left[\delta_{16}\left(\beta_{2(\mathrm{x})}-1\right)-\left(\lambda_{22}-1\right)\right]$ & $\gamma S_{\mathrm{y}}^{4}\left[\left(\beta_{2(\mathrm{y})}-1\right)+\delta_{16}^{2}\left(\beta_{2(\mathrm{x})}-1\right)-2 \delta_{16}\left(\lambda_{22}-1\right)\right]$ \\
\hline
\end{tabular}




\begin{tabular}{|c|c|c|}
\hline $\begin{array}{l}\hat{S}_{17}^{2}=s_{y}^{2}\left[\frac{S_{x}^{2}+D_{6}}{S_{x}^{2}+D_{6}}\right] \\
\text { Subramani and } \\
\text { Kumarapandiyan } \\
\text { (2012c) }\end{array}$ & $\gamma S_{y}^{2} \delta_{17}\left[\delta_{17}\left(\beta_{2(\mathrm{x})}-1\right)-\left(\lambda_{22}-1\right)\right]$ & $\gamma S_{\mathrm{y}}^{4}\left[\left(\beta_{2(\mathrm{y})}-1\right)+\delta_{17}^{2}\left(\beta_{2(\mathrm{x})}-1\right)-2 \delta_{17}\left(\lambda_{22}-1\right)\right]$ \\
\hline $\begin{array}{l}\hat{\mathrm{S}}_{18}^{2}=\mathrm{s}_{\mathrm{y}}^{2}\left[\frac{\mathrm{S}_{\mathrm{x}}^{2}+\mathrm{D}_{7}}{\mathrm{~s}_{\mathrm{x}}^{2}+\mathrm{D}_{7}}\right] \\
\text { Subramani and } \\
\text { Kumarapandiyan } \\
\text { (2012c) }\end{array}$ & $\gamma S_{\mathrm{y}}^{2} \delta_{18}\left[\delta_{18}\left(\beta_{2(\mathrm{x})}-1\right)-\left(\lambda_{22}-1\right)\right]$ & $\gamma S_{\mathrm{y}}^{4}\left[\left(\beta_{2(\mathrm{y})}-1\right)+\delta_{18}^{2}\left(\beta_{2(\mathrm{x})}-1\right)-2 \delta_{18}\left(\lambda_{22}-1\right)\right]$ \\
\hline $\begin{array}{l}\hat{S}_{19}^{2}=\mathrm{s}_{\mathrm{y}}^{2}\left[\frac{\mathrm{S}_{\mathrm{x}}^{2}+\mathrm{D}_{8}}{\mathrm{~s}_{\mathrm{x}}^{2}+\mathrm{D}_{8}}\right] \\
\text { Subramani and } \\
\text { Kumarapandiyan } \\
\text { (2012c) }\end{array}$ & $\gamma S_{\mathrm{y}}^{2} \delta_{19}\left[\delta_{19}\left(\beta_{2(\mathrm{x})}-1\right)-\left(\lambda_{22}-1\right)\right]$ & $\gamma S_{\mathrm{y}}^{4}\left[\left(\beta_{2(\mathrm{y})}-1\right)+\delta_{19}^{2}\left(\beta_{2(\mathrm{x})}-1\right)-2 \delta_{1 \circ}\left(\lambda_{22}-1\right)\right]$ \\
\hline $\begin{array}{l}\hat{S}_{20}^{2}=s_{y}^{2}\left[\frac{S_{x}^{2}+D_{9}}{s_{x}^{2}+D_{9}}\right] \\
\text { Subramani and } \\
\text { Kumarapandiyan } \\
\text { (2012c) }\end{array}$ & $\gamma S_{y}^{2} \delta_{20}\left[\delta_{20}\left(\beta_{2(\mathrm{x})}-1\right)-\left(\lambda_{22}-1\right)\right]$ & $\gamma S_{\mathrm{y}}^{4}\left[\left(\beta_{2(\mathrm{y})}-1\right)+\delta_{20}^{2}\left(\beta_{2(\mathrm{x})}-1\right)-2 \delta_{20}\left(\lambda_{22}-1\right)\right]$ \\
\hline $\begin{array}{l}\hat{S}_{21}^{2}=s_{y}^{2}\left[\frac{S_{x}^{2}+D_{10}}{s_{x}^{2}+D_{10}}\right] \\
\text { Subramani and } \\
\text { Kumarapandiyan } \\
\text { (2012c) }\end{array}$ & $\gamma S_{y}^{2} \delta_{21}\left[\delta_{21}\left(\beta_{2(\mathrm{x})}-1\right)-\left(\lambda_{22}-1\right)\right]$ & $\gamma S_{\mathrm{y}}^{4}\left[\left(\beta_{2(\mathrm{y})}-1\right)+\delta_{21}^{2}\left(\beta_{2(\mathrm{x})}-1\right)-2 \delta_{21}\left(\lambda_{22}-1\right)\right]$ \\
\hline $\begin{array}{l}\hat{\mathrm{S}}_{22}^{2} \\
=\mathrm{s}_{\mathrm{y}}^{2}\left[\frac{\beta_{2(\mathrm{x})} S_{\mathrm{x}}^{2}+\mathrm{C}_{\mathrm{x}}}{\beta_{2(\mathrm{x})} s_{\mathrm{x}}^{2}+\mathrm{C}_{\mathrm{x}}}\right] \\
\text { Kadilar and Cingi } \\
\text { (2006b) }\end{array}$ & $\gamma S_{y}^{2} \delta_{22}\left[\delta_{22}\left(\beta_{2(x)}-1\right)-\left(\lambda_{22}-1\right)\right]$ & $\gamma S_{\mathrm{y}}^{4}\left[\left(\beta_{2(\mathrm{y})}-1\right)+\delta_{22}^{2}\left(\beta_{2(\mathrm{x})}-1\right)-2 \delta_{22}\left(\lambda_{22}-1\right)\right]$ \\
\hline $\begin{array}{l}\hat{\mathrm{S}}_{23}^{2} \\
=\mathrm{s}_{\mathrm{y}}^{2}\left[\frac{\mathrm{C}_{\mathrm{x}} S_{\mathrm{x}}^{2}+\beta_{2(\mathrm{x})}}{\mathrm{C}_{\mathrm{x}} \mathrm{S}_{\mathrm{x}}^{2}+\beta_{2(\mathrm{x})}}\right] \\
\text { Kadilar and Cingi } \\
\text { (2006b) }\end{array}$ & $\gamma S_{y}^{2} \delta_{23}\left[\delta_{23}\left(\beta_{2(x)}-1\right)-\left(\lambda_{22}-1\right)\right]$ & $\gamma S_{\mathrm{y}}^{4}\left[\left(\beta_{2(\mathrm{y})}-1\right)+\delta_{23}^{2}\left(\beta_{2(\mathrm{x})}-1\right)-2 \delta_{23}\left(\lambda_{22}-1\right)\right]$ \\
\hline $\begin{array}{l}\hat{S}_{24}^{2} \\
=s_{y}^{2}\end{array}\left[\frac{\beta_{1(x)} S_{x}^{2}+C_{x}}{\beta_{1(x)} s_{x}^{2}+C_{x}}\right]$ & $\gamma S_{y}^{2} \delta_{24}\left[\delta_{24}\left(\beta_{2(x)}-1\right)-\left(\lambda_{22}-1\right)\right]$ & $\gamma S_{\mathrm{y}}^{4}\left[\left(\beta_{2(\mathrm{y})}-1\right)+\delta_{24}^{2}\left(\beta_{2(\mathrm{x})}-1\right)-2 \delta_{24}\left(\lambda_{22}-1\right)\right]$ \\
\hline $\begin{array}{l}\hat{S}_{25}^{2} \\
=s_{y}^{2}\left[\frac{C_{x} S_{x}^{2}+\beta_{1(x)}}{C_{x} S_{x}^{2}+\beta_{1(x)}}\right]\end{array}$ & $\gamma S_{y}^{2} \delta_{25}\left[\delta_{25}\left(\beta_{2(\mathrm{x})}-1\right)-\left(\lambda_{22}-1\right)\right]$ & $\gamma S_{\mathrm{y}}^{4}\left[\left(\beta_{2(\mathrm{y})}-1\right)+\delta_{25}^{2}\left(\beta_{2(\mathrm{x})}-1\right)-2 \delta_{25}\left(\lambda_{22}-1\right)\right]$ \\
\hline
\end{tabular}




\begin{tabular}{|c|c|c|}
\hline$\widehat{S}_{26}^{2}=s_{y}^{2}\left[\frac{\rho S_{x}^{2}+C_{x}}{\rho s_{x}^{2}+C_{x}}\right]$ & $\gamma S_{\mathrm{y}}^{2} \delta_{26}\left[\delta_{26}\left(\beta_{2(\mathrm{x})}-1\right)-\left(\lambda_{22}-1\right)\right]$ & $\gamma S_{\mathrm{y}}^{4}\left[\left(\beta_{2(\mathrm{y})}-1\right)+\delta_{26}^{2}\left(\beta_{2(\mathrm{x})}-1\right)-2 \delta_{26}\left(\lambda_{22}-1\right)\right]$ \\
\hline$\widehat{S}_{27}^{2}=s_{y}^{2}\left[\frac{C_{x} S_{x}^{2}+\rho}{C_{x} s_{x}^{2}+\rho}\right]$ & $\gamma S_{y}^{2} \delta_{27}\left[\delta_{27}\left(\beta_{2(x)}-1\right)-\left(\lambda_{22}-1\right)\right]$ & $\gamma S_{y}^{4}\left[\left(\beta_{2(y)}-1\right)+\delta_{27}^{2}\left(\beta_{2(x)}-1\right)-2 \delta_{27}\left(\lambda_{22}-1\right)\right]$ \\
\hline$\widehat{\mathrm{S}}_{28}^{2}=\mathrm{s}_{\mathrm{y}}^{2}\left[\frac{\mathrm{S}_{\mathrm{x}} \mathrm{S}_{\mathrm{x}}^{2}+\mathrm{C}_{\mathrm{x}}}{\mathrm{S}_{\mathrm{x}} \mathrm{s}_{\mathrm{x}}^{2}+\mathrm{C}_{\mathrm{x}}}\right]$ & $\gamma S_{y}^{2} \delta_{28}\left[\delta_{28}\left(\beta_{2(x)}-1\right)-\left(\lambda_{22}-1\right)\right]$ & $\gamma S_{\mathrm{y}}^{4}\left[\left(\beta_{2(\mathrm{y})}-1\right)+\delta_{28}^{2}\left(\beta_{2(\mathrm{x})}-1\right)-2 \delta_{28}\left(\lambda_{22}-1\right)\right]$ \\
\hline$\widehat{\mathrm{S}}_{29}^{2}=\mathrm{s}_{\mathrm{y}}^{2}\left[\frac{\mathrm{C}_{\mathrm{x}} \mathrm{S}_{\mathrm{x}}^{2}+\mathrm{S}_{\mathrm{x}}}{\mathrm{C}_{\mathrm{x}} \mathrm{s}_{\mathrm{x}}^{2}+\mathrm{S}_{\mathrm{x}}}\right]$ & $\gamma S_{y}^{2} \delta_{29}\left[\delta_{29}\left(\beta_{2(\mathrm{x})}-1\right)-\left(\lambda_{22}-1\right)\right]$ & $\gamma S_{y}^{4}\left[\left(\beta_{2(y)}-1\right)+\delta_{29}^{2}\left(\beta_{2(x)}-1\right)-2 \delta_{29}\left(\lambda_{22}-1\right)\right]$ \\
\hline$\hat{S}_{30}^{2}=s_{y}^{2}\left[\frac{M_{d} S_{x}^{2}+C_{x}}{M_{d} s_{x}^{2}+C_{x}}\right]$ & $\gamma S_{y}^{2} \delta_{30}\left[\delta_{30}\left(\beta_{2(x)}-1\right)-\left(\lambda_{22}-1\right)\right]$ & $\gamma S_{\mathrm{y}}^{4}\left[\left(\beta_{2(\mathrm{y})}-1\right)+\delta_{30}^{2}\left(\beta_{2(\mathrm{x})}-1\right)-2 \delta_{30}\left(\lambda_{22}-1\right)\right]$ \\
\hline $\begin{array}{l}\widehat{S}_{31}^{2}=s_{y}^{2}\left[\frac{C_{x} S_{x}^{2}+M_{d}}{C_{x} s_{x}^{2}+M_{d}}\right] \\
\text { Subramani and } \\
\text { Kumarapandiyan } \\
\text { (2013) }\end{array}$ & $\gamma S_{y}^{2} \delta_{31}\left[\delta_{31}\left(\beta_{2(x)}-1\right)-\left(\lambda_{22}-1\right)\right]$ & $\gamma S_{\mathrm{y}}^{4}\left[\left(\beta_{2(\mathrm{y})}-1\right)+\delta_{31}^{2}\left(\beta_{2(\mathrm{x})}-1\right)-2 \delta_{31}\left(\lambda_{22}-1\right)\right]$ \\
\hline $\begin{array}{l}\hat{S}_{32}^{2} \\
=s_{y}^{2}\left[\frac{\beta_{1(x)} S_{x}^{2}+\beta_{2(x)}}{\beta_{1(x)} s_{x}^{2}+\beta_{2(x)}}\right]\end{array}$ & $\gamma S_{y}^{2} \delta_{32}\left[\delta_{32}\left(\beta_{2(\mathrm{x})}-1\right)-\left(\lambda_{22}-1\right)\right]$ & $\gamma S_{\mathrm{y}}^{4}\left[\left(\beta_{2(\mathrm{y})}-1\right)+\delta_{32}^{2}\left(\beta_{2(\mathrm{x})}-1\right)-2 \delta_{32}\left(\lambda_{22}-1\right)\right]$ \\
\hline $\begin{array}{l}\hat{S}_{33}^{2} \\
=s_{y}^{2}\left[\frac{\beta_{2(x)} S_{x}^{2}+\beta_{1(x)}}{\beta_{2(x)} s_{x}^{2}+\beta_{1(x)}}\right]\end{array}$ & $\gamma S_{\mathrm{y}}^{2} \delta_{33}\left[\delta_{33}\left(\beta_{2(\mathrm{x})}-1\right)-\left(\lambda_{22}-1\right)\right]$ & $\gamma S_{\mathrm{y}}^{4}\left[\left(\beta_{2(\mathrm{y})}-1\right)+\delta_{33}^{2}\left(\beta_{2(\mathrm{x})}-1\right)-2 \delta_{33}\left(\lambda_{22}-1\right)\right]$ \\
\hline $\begin{array}{l}\hat{S}_{34}^{2} \\
=s_{y}^{2}\left[\frac{\rho S_{x}^{2}+\beta_{2(x)}}{\rho s_{x}^{2}+\beta_{2(x)}}\right]\end{array}$ & $\gamma S_{y}^{2} \delta_{34}\left[\delta_{34}\left(\beta_{2(x)}-1\right)-\left(\lambda_{22}-1\right)\right]$ & $\gamma S_{\mathrm{y}}^{4}\left[\left(\beta_{2(\mathrm{y})}-1\right)+\delta_{34}^{2}\left(\beta_{2(\mathrm{x})}-1\right)-2 \delta_{34}\left(\lambda_{22}-1\right)\right]$ \\
\hline
\end{tabular}




\begin{tabular}{|c|c|c|}
\hline$=s_{y}^{2}\left[\frac{\beta_{2(x)}^{2} S_{x}^{2}+\rho}{\beta_{2(x)} S_{x}^{2}+\rho}\right]$ & $\gamma S_{y}^{2} \delta_{35}\left[\delta_{35}\left(\beta_{2(x)}-1\right)-\left(\lambda_{22}-1\right)\right]$ & $\gamma S_{y}^{4}\left[\left(\beta_{2(y)}-1\right)+\delta_{35}^{2}\left(\beta_{2(x)}-1\right)-2 \delta_{35}\left(\lambda_{22}-1\right)\right]$ \\
\hline $\begin{array}{l}\hat{S}_{36}^{2} \\
=s_{y}^{2}\end{array}\left[\frac{S_{x} S_{x}^{2}+\beta_{2(x)}}{S_{x} S_{x}^{2}+\beta_{2(x)}}\right]$ & $\gamma S_{y}^{2} \delta_{36}\left[\delta_{36}\left(\beta_{2(x)}-1\right)-\left(\lambda_{22}-1\right)\right]$ & $\gamma S_{y}^{4}\left[\left(\beta_{2(y)}-1\right)+\delta_{36}^{2}\left(\beta_{2(x)}-1\right)-2 \delta_{36}\left(\lambda_{22}-1\right)\right]$ \\
\hline $\begin{array}{l}\hat{S}_{37}^{2} \\
=s_{y}^{2}\end{array}\left[\frac{\beta_{2(x)} S_{x}^{2}+S_{x}}{\beta_{2(x)} S_{x}^{2}+S_{x}}\right]$ & $\gamma S_{y}^{2} \delta_{37}\left[\delta_{37}\left(\beta_{2(x)}-1\right)-\left(\lambda_{22}-1\right)\right]$ & $\gamma S_{y}^{4}\left[\left(\beta_{2(y)}-1\right)+\delta_{37}^{2}\left(\beta_{2(x)}-1\right)-2 \delta_{37}\left(\lambda_{22}-1\right)\right]$ \\
\hline $\begin{array}{l}\hat{S}_{38}^{2} \\
=s_{y}^{2}\end{array}\left[\frac{M_{d} S_{x}^{2}+\beta_{2(x)}}{M_{d} S_{x}^{2}+\beta_{2(x)}}\right]$ & $\gamma S_{y}^{2} \delta_{38}\left[\delta_{38}\left(\beta_{2(x)}-1\right)-\left(\lambda_{22}-1\right)\right]$ & $\gamma S_{y}^{4}\left[\left(\beta_{2(y)}-1\right)+\delta_{38}^{2}\left(\beta_{2(x)}-1\right)-2 \delta_{38}\left(\lambda_{22}-1\right)\right]$ \\
\hline $\left.\begin{array}{l}\hat{S}_{39}^{2} \\
=s_{y}^{2}\end{array} \frac{\beta_{2(x)} S_{x}^{2}+M_{d}}{\beta_{2(x)} s_{x}^{2}+M_{d}}\right]$ & $\gamma S_{y}^{2} \delta_{39}\left[\delta_{39}\left(\beta_{2(x)}-1\right)-\left(\lambda_{22}-1\right)\right]$ & $\gamma S_{y}^{4}\left[\left(\beta_{2(y)}-1\right)+\delta_{39}^{2}\left(\beta_{2(x)}-1\right)-2 \delta_{39}\left(\lambda_{22}-1\right)\right]$ \\
\hline $\begin{array}{l}\hat{S}_{40}^{2} \\
=s_{y}^{2}\end{array}\left[\frac{\rho S_{x}^{2}+\beta_{1(x)}}{\rho S_{x}^{2}+\beta_{1(x)}}\right]$ & $\gamma S_{y}^{2} \delta_{40}\left[\delta_{40}\left(\beta_{2(x)}-1\right)-\left(\lambda_{22}-1\right)\right]$ & $\gamma S_{y}^{4}\left[\left(\beta_{2(y)}-1\right)+\delta_{40}^{2}\left(\beta_{2(x)}-1\right)-2 \delta_{40}\left(\lambda_{22}-1\right)\right]$ \\
\hline $\begin{array}{l}\hat{S}_{41}^{2} \\
=s_{y}^{2}\end{array}\left[\frac{\beta_{1(x)} S_{x}^{2}+\rho}{\beta_{1(x)} S_{x}^{2}+\rho}\right]$ & $\gamma S_{y}^{2} \delta_{41}\left[\delta_{41}\left(\beta_{2(x)}-1\right)-\left(\lambda_{22}-1\right)\right]$ & $\gamma S_{y}^{4}\left[\left(\beta_{2(y)}-1\right)+\delta_{41}^{2}\left(\beta_{2(x)}-1\right)-2 \delta_{41}\left(\lambda_{22}-1\right)\right]$ \\
\hline $\begin{array}{l}\widehat{S}_{42}^{2} \\
=s_{y}^{2}\end{array}\left[\frac{S_{X} S_{x}^{2}+\beta_{1(x)}}{S_{x} S_{X}^{2}+\beta_{1(x)}}\right]$ & $\gamma S_{y}^{2} \delta_{42}\left[\delta_{42}\left(\beta_{2(x)}-1\right)-\left(\lambda_{22}-1\right)\right]$ & $\gamma S_{y}^{4}\left[\left(\beta_{2(y)}-1\right)+\delta_{42}^{2}\left(\beta_{2(x)}-1\right)-2 \delta_{42}\left(\lambda_{22}-1\right)\right]$ \\
\hline$=s_{y}^{2}\left[\frac{\beta_{1(x)}^{2} S_{x}^{2}+S_{x}}{\beta_{1(x)} s_{x}^{2}+S_{x}}\right]$ & $\gamma S_{y}^{2} \delta_{43}\left[\delta_{43}\left(\beta_{2(x)}-1\right)-\left(\lambda_{22}-1\right)\right]$ & $\gamma S_{y}^{4}\left[\left(\beta_{2(y)}-1\right)+\delta_{43}^{2}\left(\beta_{2(x)}-1\right)-2 \delta_{43}\left(\lambda_{22}-1\right)\right]$ \\
\hline
\end{tabular}




\begin{tabular}{|c|c|c|}
\hline $\begin{array}{l}\widehat{S}_{44}^{2} \\
=s_{y}^{2}\left[\frac{M_{d} S_{x}^{2}+\beta_{1(x)}}{M_{d} s_{x}^{2}+\beta_{1(x)}}\right]\end{array}$ & $\gamma S_{\mathrm{y}}^{2} \delta_{44}\left[\delta_{44}\left(\beta_{2(\mathrm{x})}-1\right)-\left(\lambda_{22}-1\right)\right]$ & $\gamma S_{\mathrm{y}}^{4}\left[\left(\beta_{2(\mathrm{y})}-1\right)+\delta_{44}^{2}\left(\beta_{2(\mathrm{x})}-1\right)-2 \delta_{44}\left(\lambda_{22}-1\right)\right]$ \\
\hline $\begin{array}{l}\widehat{S}_{45}^{2} \\
=s_{y}^{2}\left[\frac{\beta_{1(\mathrm{x})} S_{\mathrm{x}}^{2}+\mathrm{M}_{\mathrm{d}}}{\beta_{1(\mathrm{x})} s_{\mathrm{x}}^{2}+\mathrm{M}_{\mathrm{d}}}\right]\end{array}$ & $\gamma S_{y}^{2} \delta_{45}\left[\delta_{45}\left(\beta_{2(\mathrm{x})}-1\right)-\left(\lambda_{22}-1\right)\right]$ & $\gamma S_{\mathrm{y}}^{4}\left[\left(\beta_{2(\mathrm{y})}-1\right)+\delta_{45}^{2}\left(\beta_{2(\mathrm{x})}-1\right)-2 \delta_{45}\left(\lambda_{22}-1\right)\right]$ \\
\hline$\widehat{S}_{46}^{2}=s_{y}^{2}\left[\frac{S_{x} S_{x}^{2}+\rho}{S_{x} s_{x}^{2}+\rho}\right]$ & $\gamma S_{\mathrm{y}}^{2} \delta_{46}\left[\delta_{46}\left(\beta_{2(\mathrm{x})}-1\right)-\left(\lambda_{22}-1\right)\right]$ & $\gamma S_{y}^{4}\left[\left(\beta_{2(y)}-1\right)+\delta_{46}^{2}\left(\beta_{2(x)}-1\right)-2 \delta_{46}\left(\lambda_{22}-1\right)\right]$ \\
\hline$\widehat{S}_{47}^{2}=\mathrm{s}_{\mathrm{y}}^{2}\left[\frac{\rho \mathrm{S}_{\mathrm{x}}^{2}+\mathrm{S}_{\mathrm{x}}}{\rho \mathrm{s}_{\mathrm{x}}^{2}+\mathrm{S}_{\mathrm{x}}}\right]$ & $\gamma S_{y}^{2} \delta_{47}\left[\delta_{47}\left(\beta_{2(x)}-1\right)-\left(\lambda_{22}-1\right)\right]$ & $\gamma S_{y}^{4}\left[\left(\beta_{2(y)}-1\right)+\delta_{47}^{2}\left(\beta_{2(x)}-1\right)-2 \delta_{47}\left(\lambda_{22}-1\right)\right]$ \\
\hline$\widehat{S}_{48}^{2}=s_{y}^{2}\left[\frac{M_{d} S_{x}^{2}+\rho}{S_{x} S_{x}^{2}+\rho}\right]$ & $\gamma S_{y}^{2} \delta_{48}\left[\delta_{48}\left(\beta_{2(\mathrm{x})}-1\right)-\left(\lambda_{22}-1\right)\right]$ & $\gamma S_{y}^{4}\left[\left(\beta_{2(y)}-1\right)+\delta_{48}^{2}\left(\beta_{2(x)}-1\right)-2 \delta_{48}\left(\lambda_{22}-1\right)\right]$ \\
\hline$\widehat{S}_{49}^{2}=s_{y}^{2}\left[\frac{\rho S_{x}^{2}+M_{d}}{\rho s_{x}^{2}+M_{d}}\right]$ & $\gamma S_{y}^{2} \delta_{49}\left[\delta_{49}\left(\beta_{2(\mathrm{x})}-1\right)-\left(\lambda_{22}-1\right)\right]$ & $\gamma S_{y}^{4}\left[\left(\beta_{2(y)}-1\right)+\delta_{49}^{2}\left(\beta_{2(x)}-1\right)-2 \delta_{49}\left(\lambda_{22}-1\right)\right]$ \\
\hline$\widehat{S}_{50}^{2}=s_{y}^{2}\left[\frac{M_{d} S_{x}^{2}+S_{x}}{M_{d} s_{x}^{2}+S_{x}}\right]$ & $\gamma S_{y}^{2} \delta_{50}\left[\delta_{50}\left(\beta_{2(\mathrm{x})}-1\right)-\left(\lambda_{22}-1\right)\right]$ & $\gamma S_{\mathrm{y}}^{4}\left[\left(\beta_{2(\mathrm{y})}-1\right)+\delta_{50}^{2}\left(\beta_{2(\mathrm{x})}-1\right)-2 \delta_{50}\left(\lambda_{22}-1\right)\right]$ \\
\hline$\widehat{S}_{51}^{2}=s_{y}^{2}\left[\frac{S_{x} S_{x}^{2}+M_{d}}{S_{x} s_{x}^{2}+M_{d}}\right]$ & $\gamma S_{y}^{2} \delta_{51}\left[\delta_{51}\left(\beta_{2(\mathrm{x})}-1\right)-\left(\lambda_{22}-1\right)\right]$ & $\gamma S_{\mathrm{y}}^{4}\left[\left(\beta_{2(\mathrm{y})}-1\right)+\delta_{51}^{2}\left(\beta_{2(\mathrm{x})}-1\right)-2 \delta_{51}\left(\lambda_{22}-1\right)\right]$ \\
\hline
\end{tabular}

$$
\begin{aligned}
& \text { where } \delta_{i}=\frac{S_{x}^{2}}{S_{x}^{2}+\omega_{i}} ; i=1,2,3, \ldots, 51 ; \omega_{1}=C_{x}, \omega_{2}=\beta_{2(x)}, \omega_{3}=\beta_{1(x)}, \omega_{4}=\rho, \\
& \omega_{5}=S_{x}, \omega_{6}=M_{d}, \omega_{7}=Q_{1}, \omega_{8}=Q_{3}, \omega_{9}=Q_{r}, \omega_{10}=Q_{d}, \omega_{11}=Q_{a}, \omega_{12}=D_{1}, \\
& \omega_{13}=D_{2}, \omega_{14}=D_{3}, \omega_{15}=D_{4}, \omega_{16}=D_{5}, \omega_{17}=D_{6}, \omega_{18}=D_{7}, \omega_{19}=D_{8}, \omega_{20}=D_{9},
\end{aligned}
$$




$$
\begin{aligned}
& \omega_{21}=D_{10}, \omega_{22}=\frac{C_{x}}{\beta_{2(x)}}, \omega_{23}=\frac{\beta_{2(x)}}{C_{x}}, \omega_{24}=\frac{C_{x}}{\beta_{1(x)}}, \omega_{25}=\frac{\beta_{1(x)}}{C_{x}}, \omega_{26}=\frac{C_{x}}{\rho}, \omega_{27}=\frac{\rho}{C_{x}} \\
& \omega_{28}=\frac{C_{x}}{S_{x}}, \omega_{29}=\frac{S_{x}}{C_{x}}, \omega_{30}=\frac{C_{x}}{M_{d}}, \omega_{31}=\frac{M_{d}}{C_{x}}, \omega_{32}=\frac{\beta_{2(x)}}{\beta_{1(x)}}, \omega_{33}=\frac{\beta_{1(x)}}{\beta_{2(x)}}, \omega_{34}=\frac{\beta_{2(x)}}{\rho} \\
& \omega_{35}=\frac{\rho}{\beta_{2(x)}}, \omega_{36}=\frac{\beta_{2(x)}}{S_{x}}, \omega_{37}=\frac{S_{x}}{\beta_{2(x)}}, \omega_{38}=\frac{\beta_{2(x)}}{M_{d}}, \omega_{39}=\frac{M_{d}}{\beta_{2(x)}}, \omega_{40}=\frac{\beta_{1(x)}}{\rho}, \omega_{41}=\frac{\rho}{\beta_{1(x)}} \\
& \omega_{42}=\frac{\beta_{1(x)}}{S_{x}}, \omega_{43}=\frac{S_{x}}{\beta_{1(x)}}, \omega_{44}=\frac{\beta_{1(x)}}{M_{d}}, \omega_{45}=\frac{M_{d}}{\beta_{1(x)}}, \omega_{46}=\frac{\rho}{S_{x}}, \omega_{47}=\frac{S_{x}}{\rho}, \omega_{48} \\
& =\frac{\beta_{1(x)}}{M_{d}}, \\
& \omega_{49}=\frac{M_{d}}{\beta_{1(x)}}, \omega_{50}=\frac{S_{x}}{M_{d}} \text { and } \omega_{51}=\frac{M_{d}}{S_{x}}
\end{aligned}
$$

Upadhyaya and Singh (2001) have suggested the following modified ratio type variance estimator using the population mean of the auxiliary variable together with its bias and mean squared error are given below:

$$
\begin{aligned}
& \widehat{\mathrm{S}}_{52}^{2}=\mathrm{s}_{\mathrm{y}}^{2}\left[\frac{\overline{\mathrm{x}}}{\overline{\mathrm{x}}}\right] \\
& \mathrm{B}\left(\widehat{\mathrm{S}}_{52}^{2}\right)=\gamma \mathrm{S}_{\mathrm{y}}^{2}\left[\mathrm{C}_{\mathrm{x}}^{2}-\lambda_{21} \mathrm{C}_{\mathrm{x}}\right] \\
& \operatorname{MSE}\left(\widehat{\mathrm{S}}_{52}^{2}\right)=\gamma \mathrm{S}_{\mathrm{y}}^{4}\left[\left(\beta_{2(\mathrm{y})}-1\right)+\mathrm{C}_{\mathrm{x}}^{2}-2 \lambda_{21} C_{\mathrm{x}}\right] \text { where } \lambda_{21}=\frac{\mu_{21}}{\mu_{20} \sqrt{\mu_{02}}}
\end{aligned}
$$

The modified ratio type variance estimators discussed above are biased but have smaller mean squared errors compared to the traditional ratio type variance estimator suggested by Isaki (1983) under certain conditions. The list of estimators given in Table 1 uses the known values of the parameters and their linear combinations and improved the traditional ratio type estimator. In this paper an attempt has been made to modify the ratio type variance estimator suggested by Upadhyaya and Singh (2001) using known parameters of the auxiliary variable and its linear combination. The materials of the present study are arranged as given below. The proposed estimators using known parameters of the auxiliary variable are presented in section 2 where as the conditions in which the proposed estimators perform better than the traditional and existing modified estimators are derived in section 3. The 
performance of the proposed estimators with that of the traditional and existing modified estimators are assessed for certain natural populations in section 4 and the conclusion is presented in section 5 .

\section{PROPOSED ESTIMATORS}

In this section we have suggested a class of modified ratio type variance estimators using the known parameters of the auxiliary variable for estimating the population variance of the study variable $Y$. The proposed class of modified ratio type variance estimators $\widehat{S}_{\mathrm{p}_{\mathrm{i}}}^{2}, \mathrm{i}=1,2, \ldots, 51$ for estimating the population variance $\mathrm{S}_{\mathrm{y}}^{2}$ is given below:

$\widehat{\mathrm{S}}_{\mathrm{p}_{\mathrm{i}}}^{2}=\mathrm{s}_{\mathrm{y}}^{2}\left[\frac{\overline{\mathrm{X}}+\omega_{\mathrm{i}}}{\overline{\mathrm{x}}+\omega_{\mathrm{i}}}\right] ; \mathrm{i}=1,2,3, \ldots, 51$

The bias and mean squared error of the proposed estimators $\widehat{S}_{p_{i}}^{2}, i=1,2, \ldots, 51$ have been derived (see Appendix A) and are given below

$\operatorname{Bias}\left(\widehat{S}_{\mathrm{p}_{\mathrm{i}}}^{2}\right)=\frac{(1-\mathrm{f})}{\mathrm{n}} \mathrm{S}_{\mathrm{y}}^{2}\left(\theta_{\mathrm{p}_{\mathrm{i}}}^{2} \mathrm{C}_{\mathrm{x}}^{2}-\theta_{\mathrm{p}_{\mathrm{i}}} \lambda_{21} \mathrm{C}_{\mathrm{x}}\right)$

$\operatorname{MSE}\left(\widehat{\mathrm{S}}_{\mathrm{p}_{\mathrm{i}}}^{2}\right)=\frac{(1-\mathrm{f})}{\mathrm{n}} \mathrm{S}_{\mathrm{y}}^{4}\left(\left(\beta_{2(\mathrm{y})}-1\right)+\theta_{\mathrm{p}_{\mathrm{i}}}^{2} \mathrm{C}_{\mathrm{x}}^{2}-2 \theta_{\mathrm{p}_{\mathrm{i}}} \lambda_{21} \mathrm{C}_{\mathrm{x}}\right) ; \mathrm{i}=1,2,3, \ldots, 51$

where $\theta_{p_{i}}=\frac{\bar{X}}{\overline{\mathrm{X}}+\omega_{i}}$

REMARK 2.1 When the study variable $\mathrm{Y}$ and auxiliary variable $\mathrm{X}$ are negatively correlated and the population parameters of the auxiliary variable are known, the following modified product type variance estimators can be proposed:

$\widehat{\mathrm{S}}_{\mathrm{p}_{\mathrm{i}}}^{2}=\mathrm{s}_{\mathrm{y}}^{2}\left[\frac{\overline{\mathrm{x}}+\omega_{\mathrm{i}}}{\overline{\mathrm{x}}+\omega_{\mathrm{i}}}\right] \mathrm{i}=1,2,3, \ldots, 51$

\section{EFFICIENCY OF THE PROPOSED ESTIMATORS}

As we mentioned earlier the mean squared error of the traditional ratio type variance estimator $\widehat{\mathbf{S}}_{\mathbf{r}}^{2}$ is given below:

$$
\operatorname{MSE}\left(\widehat{S}_{\mathrm{R}}^{2}\right)=\gamma \mathrm{S}_{\mathrm{y}}^{4}\left[\left(\beta_{2(\mathrm{y})}-1\right)+\left(\beta_{2(\mathrm{x})}-1\right)-2\left(\lambda_{22}-1\right)\right]
$$

The mean squared error of the modified ratio type variance estimators $\hat{\mathbf{S}}_{\mathbf{i}}^{2}$ given in table 1 are represented in single class as given below: 
$\operatorname{MSE}\left(\hat{\mathrm{S}}_{\mathrm{i}}^{2}\right)=\gamma \mathrm{S}_{\mathrm{y}}^{4}\left[\left(\beta_{2(\mathrm{y})}-1\right)+\delta_{\mathrm{i}}^{2}\left(\beta_{2(\mathrm{x})}-1\right)-2 \delta_{\mathrm{i}}\left(\lambda_{22}-1\right)\right] ; \mathrm{i}=1,2,3, \ldots, 51$

The mean squared error of the modified ratio type variance estimators $\widehat{\mathbf{S}}_{\mathbf{5 2}}^{2}$ suggested by upadhyaya and singh (2001) is given below:

$$
\operatorname{MSE}\left(\widehat{S}_{52}^{2}\right)=\gamma S_{y}^{4}\left[\left(\beta_{2(y)}-1\right)+C_{x}^{2}-2 \lambda_{21} C_{x}\right]
$$

The mean squared errors of the proposed modified ratio type variance estimators are given below:

$$
\operatorname{MSE}\left(\widehat{\mathrm{S}}_{\mathrm{p}_{\mathrm{i}}}^{2}\right)=\frac{(1-\mathrm{f})}{\mathrm{n}} \mathrm{S}_{\mathrm{y}}^{4}\left(\left(\beta_{2(\mathrm{y})}-1\right)+\theta_{\mathrm{p}_{\mathrm{i}}}^{2} \mathrm{C}_{\mathrm{x}}^{2}-2 \theta_{\mathrm{p}_{\mathrm{i}}} \lambda_{21} \mathrm{C}_{\mathrm{x}}\right) ; \mathrm{i}=1,2,3, \ldots, 51
$$

From the expressions given in (3.1) and (3.4) we have derived (see Appendix B) the condition for which the proposed estimators $\widehat{\mathbf{S}}_{\mathbf{p}_{\mathbf{i}}}^{2}, \mathbf{i}=\mathbf{1}, \mathbf{2}, \mathbf{3}, \ldots, \mathbf{5 1}$ are more efficient than the traditional ratio type variance estimator and it is given below:

$\operatorname{MSE}\left(\widehat{\mathrm{S}}_{\mathrm{p}_{\mathrm{i}}}^{2}\right)<\operatorname{MSE}\left(\widehat{\mathrm{S}}_{\mathrm{R}}^{2}\right)$ if $\theta_{\mathrm{p}_{\mathrm{i}}} \leq \frac{\lambda_{21}+\left(\left(\beta_{2(\mathrm{x})}-1\right)-2\left(\lambda_{22}-1\right)+\lambda_{21}^{2}\right)^{\frac{1}{2}}}{\mathrm{C}_{\mathrm{x}}}$

From the expressions given in (3.2) and (3.4) we have derived (see Appendix C) the conditions for which the proposed estimators $\mathbf{S}_{\mathbf{p}_{\mathbf{i}}}^{2}, \mathbf{i}=\mathbf{1}, \mathbf{2}, \mathbf{3}, \ldots, \mathbf{5 1}$ are more efficient than the modified ratio type variance estimators given in table $1, \widehat{\mathbf{S}}_{\mathbf{i}}^{2} ; \mathbf{i}=$ $\mathbf{1}, \mathbf{2}, \mathbf{3}, \ldots, \mathbf{5 1}$ and are given below:

$\operatorname{MSE}\left(\widehat{\mathrm{S}}_{\mathrm{p}_{\mathrm{i}}}^{2}\right)<\operatorname{MSE}\left(\widehat{\mathrm{S}}_{\mathrm{i}}^{2}\right)$ if $\theta_{\mathrm{p}_{\mathrm{i}}} \leq \frac{\lambda_{21}+\left(\delta_{\mathrm{i}}^{2}\left(\beta_{2(\mathrm{x})}-1\right)-2 \delta_{\mathrm{i}}\left(\lambda_{22}-1\right)+\lambda_{21}^{2}\right)^{\frac{1}{2}}}{\mathrm{C}_{\mathrm{x}}}$

From the expressions given in (3.3) and (3.4) we have derived (see Appendix D) the conditions for which the proposed estimators $\widehat{\mathbf{S}}_{\mathbf{p}_{\mathbf{i}}}^{2}, \mathbf{i}=\mathbf{1}, \mathbf{2}, \mathbf{3}, \ldots, \mathbf{5 1}$ are more efficient than the modified ratio type variance estimator $\widehat{\mathbf{S}}_{\mathbf{5 2}}^{2}$ and are given below:

$$
\operatorname{MSE}\left(\widehat{\mathrm{S}}_{\mathrm{p}_{\mathrm{i}}}^{2}\right) \leq \operatorname{MSE}\left(\widehat{\mathrm{S}}_{52}^{2}\right) \text { either } 2 \frac{\lambda_{21}}{\mathrm{C}_{\mathrm{x}}}-1 \leq \theta_{\mathrm{p}_{\mathrm{i}}} \leq 1 \text { (or) } 1 \leq \theta_{\mathrm{p}_{\mathrm{i}}} \leq 2 \frac{\lambda_{21}}{\mathrm{C}_{\mathrm{x}}}-1
$$




\section{NUMERICAL STUDY}

The performance of the proposed modified ratio type variance estimators listed in table 2 are assessed with that of traditional ratio type estimator and existing modified ratio type variance estimators listed for certain natural populations. The populations 1 and 2 are taken from Singh and Chaudhary (1986) given in page 177. The population parameters and the constants computed from the above populations are given below:

Population 1: Singh and Chaudhary (1986)

\begin{tabular}{|c|c|c|c|c|}
\hline $\mathrm{N}=34$ & $n=20$ & $\begin{array}{l}\bar{Y} \\
=85.6412\end{array}$ & $\bar{X}=20.8882$ & $S_{y}=73.3141$ \\
\hline$\lambda_{22}=1.1525$ & $S_{x}=15.0506$ & $C_{x}=0.7205$ & $\begin{array}{l}\beta_{2(y)} \\
=13.3666\end{array}$ & $\begin{array}{l}\beta_{2(x)} \\
=2.9123\end{array}$ \\
\hline $\begin{array}{l}\beta_{1(\mathrm{x})} \\
=0.8732\end{array}$ & $\begin{array}{l}\lambda_{21} \\
=-0.3104\end{array}$ & $M_{d}=15$ & $\mathrm{Q}_{1}=9.425$ & $Q_{3}=25.475$ \\
\hline$Q_{r}=16.05$ & $Q_{d}=16.05$ & $\mathrm{Q}_{\mathrm{a}}=17.45$ & $\mathrm{D}_{1}=7.03$ & $\mathrm{D}_{2}=7.68$ \\
\hline $\mathrm{D}_{3}=10.82$ & $\mathrm{D}_{4}=12.94$ & $D_{5}=15$ & $\mathrm{D}_{6}=22.72$ & $\mathrm{D}_{7}=25.04$ \\
\hline$D_{8}=33.56$ & $\mathrm{D}_{9}=43.61$ & $D_{10}=56.4$ & & \\
\hline
\end{tabular}

Population 2: Singh and Chaudhary (1986)

$\begin{array}{lllll}\mathrm{N}=34 & \mathrm{n}=20 & \overline{\mathrm{Y}}=85.6412 & \overline{\mathrm{X}}=19.9441 & \mathrm{~S}_{\mathrm{y}}=73.3141 \\ \lambda_{22}=1.2244 & \mathrm{~S}_{\mathrm{x}}=15.0215 & \mathrm{C}_{\mathrm{x}}=0.7532 & \beta_{2(\mathrm{y})} & \beta_{2(\mathrm{x})} \\ \beta_{1(\mathrm{x})} & \lambda_{21} & \mathrm{M}_{\mathrm{d}}=14.25 & \mathrm{Q}_{1}=9.925 & \mathrm{Q}_{3}=27.8 \\ =1.2758 & =-0.2946 & \mathrm{Q}_{\mathrm{a}} & & \\ \mathrm{Q}_{\mathrm{r}}=17.875 & \mathrm{Q}_{\mathrm{d}}=8.9375 & =18.8625 & \mathrm{D}_{1}=6.06 & \mathrm{D}_{2}=8.3 \\ \mathrm{D}_{3}=10.27 & \mathrm{D}_{4}=11.12 & \mathrm{D}_{5}=14.25 & \mathrm{D}_{6}=21.02 & \mathrm{D}_{7}=26.45 \\ \mathrm{D}_{8}=30.44 & \mathrm{D}_{9}=37.32 & \mathrm{D}_{10}=63.4 & & \end{array}$

5 .

The mean squared error of the existing and proposed modified ratio type variance estimators for the above population given below: 
Table 2 MSE(.) of the existing and proposed modified ratio type variance estimators for population 1

\begin{tabular}{|c|c|c|c|c|c|}
\hline $\begin{array}{l}\text { Existing } \\
\text { Estimator }\end{array}$ & $\operatorname{Bias}()$. & $\operatorname{MSE}()$. & $\begin{array}{l}\text { Proposed } \\
\text { Estimator } \\
\end{array}$ & $\operatorname{Bias}()$. & MSE(.) \\
\hline$\widehat{\mathrm{S}}_{1}^{2}$ & 193.4567 & 8305040.9247 & $\widehat{\mathrm{S}}_{\mathrm{p}_{1}}^{2}$ & 77.6142 & 7901409.5289 \\
\hline$\widehat{\mathrm{S}}_{2}^{2}$ & 189.6191 & 8285277.5233 & $\widehat{\mathrm{S}}_{\mathrm{p}_{2}}^{2}$ & 65.9785 & 7827023.2643 \\
\hline$\widehat{\mathrm{S}}_{3}^{2}$ & 193.1857 & 8303644.9272 & $\widehat{\mathrm{S}}_{\mathrm{p}_{3}}^{2}$ & 76.6955 & 7895569.2207 \\
\hline$\widehat{\mathrm{S}}_{4}^{2}$ & 193.9398 & 8307529.5562 & $\widehat{\mathrm{S}}_{\mathrm{p}_{4}}^{2}$ & 79.2931 & 7912069.8376 \\
\hline$\widehat{\mathrm{S}}_{5}^{2}$ & 170.2472 & 8185653.1155 & $\widehat{S}_{\mathrm{p}_{5}}^{2}$ & 33.7963 & 7614602.8707 \\
\hline$\widehat{\mathrm{S}}_{6}^{2}$ & 170.3219 & 8186036.5850 & $\widehat{\mathrm{S}}_{\mathrm{p}_{6}}^{2}$ & 33.8714 & 7615115.3167 \\
\hline$\widehat{\mathrm{S}}_{7}^{2}$ & 194.2999 & 8309384.7128 & $\widehat{\mathrm{S}}_{\mathrm{p}_{7}}^{2}$ & 80.5802 & 7920230.8059 \\
\hline$\widehat{\mathrm{S}}_{8}^{2}$ & 187.6845 & 8275317.6357 & $\widehat{\mathrm{S}}_{\mathrm{p}_{8}}^{2}$ & 61.0748 & 7795375.0862 \\
\hline$\widehat{S}_{9}^{2}$ & 193.2710 & 8304084.3017 & $\widehat{\mathrm{S}}_{\mathrm{p}_{9}}^{2}$ & 76.9829 & 7897396.7792 \\
\hline$\widehat{S}_{10}^{2}$ & 192.5865 & 8300558.7975 & $\widehat{\mathrm{S}}_{\mathrm{p}_{10}}^{2}$ & 74.7213 & 7883000.6155 \\
\hline$\widehat{S}_{11}^{2}$ & 191.8956 & 8297000.4445 & $\widehat{\mathrm{S}}_{\mathrm{p}_{11}}^{2}$ & 72.5377 & 7869069.1599 \\
\hline$\widehat{S}_{12}^{2}$ & 193.6296 & 8305931.4127 & $\widehat{\mathrm{S}}_{\mathrm{p}_{12}}^{2}$ & 78.2088 & 7905186.9248 \\
\hline$\widehat{\mathrm{S}}_{13}^{2}$ & 194.6572 & 8311225.1070 & $\widehat{\mathrm{S}}_{\mathrm{p}_{13}}^{2}$ & 81.8881 & 7928514.0560 \\
\hline$\widehat{S}_{14}^{2}$ & 161.9435 & 8143027.5287 & $\widehat{S}_{\mathrm{p}_{14}}^{2}$ & 26.7408 & 7565872.9971 \\
\hline$\widehat{\mathrm{S}}_{15}^{2}$ & 194.6569 & 8311223.6155 & $\widehat{\mathrm{S}}_{\mathrm{p}_{15}}^{2}$ & 81.8870 & 7928507.2658 \\
\hline$\widehat{\mathrm{S}}_{16}^{2}$ & 162.0399 & 8143521.8877 & $\widehat{\mathrm{S}}_{\mathrm{p}_{16}}^{2}$ & 26.8101 & 7566357.2506 \\
\hline$\widehat{S}_{17}^{2}$ & 188.8916 & 8281531.8967 & $\widehat{\mathrm{S}}_{\mathrm{p}_{17}}^{2}$ & 64.0680 & 7814716.2766 \\
\hline$\widehat{S}_{18}^{2}$ & 194.2062 & 8308901.9409 & $\widehat{\mathrm{S}}_{\mathrm{p}_{18}}^{2}$ & 80.2422 & 7918089.0587 \\
\hline$\widehat{S}_{19}^{2}$ & 183.5978 & 8254285.7975 & $\widehat{\mathrm{S}}_{\mathrm{p}_{19}}^{2}$ & 52.3462 & 7738509.3354 \\
\hline$\widehat{\mathrm{S}}_{20}^{2}$ & 194.4667 & 8310243.7205 & $\widehat{\mathrm{S}}_{\mathrm{p}_{20}}^{2}$ & 81.1867 & 7924073.4637 \\
\hline$\widehat{S}_{21}^{2}$ & 194.3964 & 8309881.4741 & $\widehat{S}_{\mathrm{p}_{21}}^{2}$ & 80.9301 & 7922448.0103 \\
\hline$\widehat{\mathrm{S}}_{22}^{2}$ & 185.7842 & 8265536.8722 & $\widehat{\mathrm{S}}_{\mathrm{p}_{22}}^{2}$ & 56.7674 & 7767404.1126 \\
\hline$\widehat{S}_{23}^{2}$ & 194.3952 & 8309875.4575 & $\widehat{\mathrm{S}}_{\mathrm{p}_{23}}^{2}$ & 80.9259 & 7922421.0747 \\
\hline$\widehat{S}_{24}^{2}$ & 185.8133 & 8265686.6605 & $\widehat{\mathrm{S}}_{\mathrm{p}_{24}}^{2}$ & 56.8299 & 7767811.3938 \\
\hline$\widehat{S}_{25}^{2}$ & 191.3000 & 8293932.9140 & $\widehat{\mathrm{S}}_{\mathrm{p}_{25}}^{2}$ & 70.7307 & 7857516.3340 \\
\hline$\widehat{S}_{26}^{2}$ & 193.8236 & 8306930.9379 & $\widehat{\mathrm{S}}_{\mathrm{p}_{26}}^{2}$ & 78.8843 & 7909475.9332 \\
\hline$\widehat{S}_{27}^{2}$ & 194.6390 & 8311131.4101 & $\widehat{\mathrm{S}}_{\mathrm{p}_{27}}^{2}$ & 81.8207 & 7928087.7466 \\
\hline$\widehat{\mathrm{S}}_{28}^{2}$ & 167.0682 & 8169328.3846 & $\widehat{\mathrm{S}}_{\mathrm{p}_{28}}^{2}$ & 30.8108 & 7594123.3077 \\
\hline$\widehat{S}_{29}^{2}$ & 194.6386 & 8311129.6027 & $\widehat{\mathrm{S}}_{\mathrm{p}_{29}}^{2}$ & 81.8194 & 7928079.5282 \\
\hline$\widehat{S}_{30}^{2}$ & 167.1514 & 8169755.4807 & $\widehat{\mathrm{S}}_{\mathrm{p}_{30}}^{2}$ & 30.8840 & 7594627.7126 \\
\hline$\widehat{\mathrm{S}}_{31}^{2}$ & 194.6895 & 8311391.6909 & $\widehat{\mathrm{S}}_{\mathrm{p}_{31}}^{2}$ & 82.0080 & 7929273.2247 \\
\hline$\widehat{\mathrm{S}}_{32}^{2}$ & 145.8871 & 8060755.6421 & $\widehat{\mathrm{S}}_{\mathrm{p}_{32}}^{2}$ & 17.9756 & 7503320.8713 \\
\hline$\widehat{S}_{33}^{2}$ & 194.6893 & 8311390.7610 & $\widehat{\mathrm{S}}_{\mathrm{p}_{33}}^{2}$ & 82.0073 & 7929268.9825 \\
\hline$\widehat{\mathrm{S}}_{34}^{2}$ & 146.0200 & 8061435.6153 & $\widehat{\mathrm{S}}_{\mathrm{p}_{34}}^{2}$ & 18.0305 & 7503722.1084 \\
\hline
\end{tabular}




\begin{tabular}{|c|c|c|c|c|c|}
\hline$\widehat{\mathrm{S}}_{35}^{2}$ & 192.9551 & 8302457.3932 & $\widehat{\mathrm{S}}_{\mathrm{p}_{35}}^{2}$ & 75.9268 & 7890677.8152 \\
\hline$\widehat{\mathrm{S}}_{36}^{2}$ & 192.9670 & 8302518.7994 & $\widehat{\mathrm{S}}_{\mathrm{p}_{36}}^{2}$ & 75.9662 & 7890929.0386 \\
\hline$\widehat{\mathrm{S}}_{37}^{2}$ & 178.8485 & 8229857.3273 & $\widehat{\mathrm{S}}_{\mathrm{p}_{37}}^{2}$ & 44.3384 & 7685618.9421 \\
\hline$\widehat{\mathrm{S}}_{38}^{2}$ & 155.8258 & 8111656.3586 & $\widehat{\mathrm{S}}_{\mathrm{p}_{38}}^{2}$ & 22.8150 & 7538189.7352 \\
\hline$\widehat{\mathrm{S}}_{39}^{2}$ & 168.7823 & 8178129.8387 & $\widehat{S}_{\mathrm{p}_{39}}^{2}$ & 32.3710 & 7604849.6035 \\
\hline$\widehat{\mathrm{S}}_{40}^{2}$ & 181.0872 & 8241370.6859 & $\widehat{\mathrm{S}}_{\mathrm{p}_{40}}^{2}$ & 47.8702 & 7709041.6847 \\
\hline$\widehat{\mathrm{S}}_{41}^{2}$ & 166.7609 & 8167750.7848 & $\widehat{\mathrm{S}}_{\mathrm{p}_{41}}^{2}$ & 30.5426 & 7592273.8712 \\
\hline$\widehat{\mathrm{S}}_{42}^{2}$ & 182.7032 & 8249683.3804 & $\widehat{\mathrm{S}}_{\mathrm{p}_{42}}^{2}$ & 50.6830 & 7727586.6369 \\
\hline$\widehat{\mathrm{S}}_{43}^{2}$ & 181.6452 & 8244240.6215 & $\widehat{\mathrm{S}}_{\mathrm{p}_{43}}^{2}$ & 48.8148 & 7715279.6791 \\
\hline$\widehat{\mathrm{S}}_{44}^{2}$ & 176.6576 & 8218593.1860 & $\widehat{\mathrm{S}}_{\mathrm{p}_{44}}^{2}$ & 41.2404 & 7664933.2877 \\
\hline$\widehat{S}_{45}^{2}$ & 173.4022 & 8201861.6358 & $\widehat{\mathrm{S}}_{\mathrm{p}_{45}}^{2}$ & 37.1913 & 7637676.2776 \\
\hline$\widehat{S}_{46}^{2}$ & 170.3219 & 8186036.5850 & $\widehat{\mathrm{S}}_{\mathrm{p}_{46}}^{2}$ & 33.8714 & 7615115.3167 \\
\hline$\widehat{\mathrm{S}}_{47}^{2}$ & 159.4592 & 8130284.6462 & $\widehat{\mathrm{S}}_{\mathrm{p}_{47}}^{2}$ & 25.0396 & 7553934.3985 \\
\hline$\widehat{\mathrm{S}}_{48}^{2}$ & 156.3915 & 8114555.7223 & $\widehat{\mathrm{S}}_{\mathrm{p}_{48}}^{2}$ & 23.1425 & 7540518.2755 \\
\hline$\widehat{\mathrm{S}}_{49}^{2}$ & 145.8315 & 8060471.0773 & $\widehat{S}_{\mathrm{p}_{49}}^{2}$ & 17.9527 & 7503153.4053 \\
\hline$\widehat{S}_{50}^{2}$ & 134.6559 & 8003341.2091 & $\widehat{\mathrm{S}}_{\mathrm{p}_{50}^{2}}^{2}$ & 14.0431 & 7474185.5247 \\
\hline$\widehat{S}_{51}^{2}$ & 122.1457 & 7939536.8330 & $\widehat{\mathrm{S}}_{\mathrm{p}_{51}}^{2}$ & 10.8871 & 7450090.5703 \\
\hline$\widehat{S}_{52}^{2}$ & 82.2071 & 7930533.2085 & & & \\
\hline$\widehat{\mathrm{S}}_{\mathrm{R}}^{2}$ & 152.5580 & 8311667.4056 & & & \\
\hline
\end{tabular}


Table 3 MSE(.) of the existing and proposed modified ratio type variance estimators for population 2

\begin{tabular}{|c|c|c|c|c|c|}
\hline $\begin{array}{l}\text { Existing } \\
\text { Estimator }\end{array}$ & $\operatorname{Bias}()$. & $\operatorname{MSE}()$. & $\begin{array}{l}\text { Proposed } \\
\text { Estimator }\end{array}$ & Bias(.) & $\operatorname{MSE}()$. \\
\hline$\widehat{\mathrm{S}}_{1}^{2}$ & 274.8748 & 8700066.3664 & $\widehat{\mathrm{S}}_{\mathrm{p}_{1}}^{2}$ & 81.9543 & 7923335.0831 \\
\hline$\widehat{\mathrm{S}}_{2}^{2}$ & 267.4800 & 8662043.5448 & $\widehat{\mathrm{S}}_{\mathrm{p}_{2}}^{2}$ & 65.2611 & 7817638.5301 \\
\hline$\widehat{\mathrm{S}}_{3}^{2}$ & 273.5533 & 8693269.2999 & $\widehat{\mathrm{S}}_{\mathrm{p}_{3}}^{2}$ & 78.5355 & 7901826.3797 \\
\hline$\widehat{\mathrm{S}}_{4}^{2}$ & 275.6578 & 8704093.4323 & $\widehat{\mathrm{S}}_{\mathrm{p}_{4}}^{2}$ & 84.0855 & 7936710.5220 \\
\hline$\widehat{\mathrm{S}}_{5}^{2}$ & 241.8679 & 8530542.5925 & $\widehat{\mathrm{S}}_{\mathrm{p}_{5}}^{2}$ & 34.4309 & 7616001.5114 \\
\hline$\widehat{\mathrm{S}}_{6}^{2}$ & 243.5012 & 8538919.0760 & $\widehat{\mathrm{S}}_{\mathrm{p}_{6}}^{2}$ & 35.6790 & 7624408.3942 \\
\hline$\widehat{\mathrm{S}}_{7}^{2}$ & 276.2783 & 8707285.4442 & $\widehat{\mathrm{S}}_{\mathrm{p}_{7}}^{2}$ & 85.8340 & 7947666.4656 \\
\hline$\widehat{\mathrm{S}}_{8}^{2}$ & 264.5267 & 8646864.7198 & $\widehat{\mathrm{S}}_{\mathrm{p}_{8}}^{2}$ & 59.9815 & 7783806.2406 \\
\hline$\widehat{S}_{9}^{2}$ & 275.2885 & 8702194.1132 & $\widehat{\mathrm{S}}_{\mathrm{p}_{9}}^{2}$ & 83.0702 & 7930341.3699 \\
\hline$\widehat{\mathrm{S}}_{10}^{2}$ & 272.5028 & 8687867.1404 & $\widehat{\mathrm{S}}_{\mathrm{p}_{10}}^{2}$ & 75.9674 & 7885626.4956 \\
\hline$\widehat{\mathrm{S}}_{11}^{2}$ & 272.5089 & 8687898.6943 & $\widehat{\mathrm{S}}_{\mathrm{p}_{11}}^{2}$ & 75.9820 & 7885718.9285 \\
\hline$\widehat{\mathrm{S}}_{12}^{2}$ & 275.2863 & 8702182.9335 & $\widehat{\mathrm{S}}_{\mathrm{p}_{12}}^{2}$ & 83.0643 & 7930304.2040 \\
\hline$\widehat{S}_{13}^{2}$ & 276.6674 & 8709286.6597 & $\widehat{\mathrm{S}}_{\mathrm{p}_{13}}^{2}$ & 86.9579 & 7954701.1868 \\
\hline$\widehat{\mathrm{S}}_{14}^{2}$ & 231.8128 & 8479004.9265 & $\widehat{\mathrm{S}}_{\mathrm{p}_{14}}^{2}$ & 27.9728 & 7571999.8359 \\
\hline$\widehat{\mathrm{S}}_{15}^{2}$ & 276.6604 & 8709250.8872 & $\widehat{\mathrm{S}}_{\mathrm{p}_{15}}^{2}$ & 86.9377 & 7954574.2911 \\
\hline$\widehat{S}_{16}^{2}$ & 233.8546 & 8489465.8908 & $\widehat{\mathrm{S}}_{\mathrm{p}_{16}}^{2}$ & 29.1347 & 7579983.9267 \\
\hline$\widehat{\mathrm{S}}_{17}^{2}$ & 269.4550 & 8672196.4026 & $\widehat{\mathrm{S}}_{\mathrm{p}_{17}}^{2}$ & 69.1858 & 7842651.4287 \\
\hline$\widehat{\mathrm{S}}_{18}^{2}$ & 275.9200 & 8705442.4919 & $\widehat{\mathrm{S}}_{\mathrm{p}_{18}}^{2}$ & 84.8179 & 7941301.8389 \\
\hline$\widehat{S}_{19}^{2}$ & 256.5008 & 8605634.9323 & $\widehat{\mathrm{S}}_{\mathrm{p}_{19}}^{2}$ & 48.4540 & 7709070.5888 \\
\hline$\widehat{\mathrm{S}}_{20}^{2}$ & 276.4897 & 8708372.8292 & $\widehat{\mathrm{S}}_{\mathrm{p}_{20}}^{2}$ & 86.4420 & 7951472.7129 \\
\hline$\widehat{S}_{21}^{2}$ & 276.1611 & 8706682.5452 & $\widehat{\mathrm{S}}_{\mathrm{p}_{21}}^{2}$ & 85.4996 & 7945572.4829 \\
\hline$\widehat{\mathrm{S}}_{22}^{2}$ & 266.7349 & 8658213.4456 & $\widehat{S}_{\mathrm{p}_{22}}^{2}$ & 63.8659 & 7808719.1725 \\
\hline$\widehat{\mathrm{S}}_{23}^{2}$ & 276.1268 & 8706506.0880 & $\widehat{\mathrm{S}}_{\mathrm{p}_{23}}^{2}$ & 85.4021 & 7944961.8077 \\
\hline$\widehat{\mathrm{S}}_{24}^{2}$ & 267.2385 & 8660802.2542 & $\widehat{\mathrm{S}}_{\mathrm{p}_{24}}^{2}$ & 64.8041 & 7814718.1590 \\
\hline$\widehat{S}_{25}^{2}$ & 269.5908 & 8672894.5544 & $\widehat{\mathrm{S}}_{\mathrm{p}_{25}}^{2}$ & 69.4685 & 7844448.5663 \\
\hline$\widehat{\mathrm{S}}_{26}^{2}$ & 275.9032 & 8705356.1073 & $\widehat{\mathrm{S}}_{\mathrm{p}_{26}}^{2}$ & 84.7708 & 7941006.1426 \\
\hline$\widehat{\mathrm{S}}_{27}^{2}$ & 276.5782 & 8708828.2800 & $\widehat{\mathrm{S}}_{\mathrm{p}_{27}}^{2}$ & 86.6986 & 7953078.3599 \\
\hline$\widehat{\mathrm{S}}_{28}^{2}$ & 248.8531 & 8566375.7830 & $\widehat{\mathrm{S}}_{\mathrm{p}_{28}}^{2}$ & 40.2619 & 7655051.1228 \\
\hline$\widehat{S}_{29}^{2}$ & 276.5665 & 8708767.7148 & $\widehat{\mathrm{S}}_{\mathrm{p}_{29}}^{2}$ & 86.6644 & 7952864.4529 \\
\hline$\widehat{S}_{30}^{2}$ & 250.1860 & 8573216.2487 & $\widehat{\mathrm{S}}_{\mathrm{p}_{30}}^{2}$ & 41.5362 & 7663513.4206 \\
\hline$\widehat{\mathrm{S}}_{31}^{2}$ & 276.7199 & 8709556.8046 & $\widehat{\mathrm{S}}_{\mathrm{p}_{31}}^{2}$ & 87.1113 & 7955660.8324 \\
\hline$\widehat{\mathrm{S}}_{32}^{2}$ & 206.6709 & 8350388.1091 & $\widehat{\mathrm{S}}_{\mathrm{p}_{32}}^{2}$ & 17.7903 & 7500315.7683 \\
\hline$\widehat{\mathrm{S}}_{33}^{2}$ & 276.7158 & 8709535.6494 & $\widehat{\mathrm{S}}_{\mathrm{p}_{33}}^{2}$ & 87.0993 & 7955585.5952 \\
\hline$\widehat{\mathrm{S}}_{34}^{2}$ & 209.6060 & 8365383.3370 & $\widehat{\mathrm{S}}_{\mathrm{p}_{34}}^{2}$ & 18.6824 & 7506746.2177 \\
\hline
\end{tabular}




\begin{tabular}{|c|c|c|c|c|c|}
\hline$\widehat{S}_{35}^{2}$ & 274.1127 & 8696146.6470 & $\widehat{\mathrm{S}}_{\mathrm{p}_{35}}^{2}$ & 79.9563 & 7910773.1951 \\
\hline$\widehat{\mathrm{S}}_{36}^{2}$ & 274.3795 & 8697518.9442 & $\widehat{\mathrm{S}}_{\mathrm{p}_{36}}^{2}$ & 80.6475 & 7915121.2084 \\
\hline$\widehat{S}_{37}^{2}$ & 252.9612 & 8587461.1638 & $\widehat{\mathrm{S}}_{\mathrm{p}_{37}}^{2}$ & 44.3855 & 7682352.0994 \\
\hline$\widehat{\mathrm{S}}_{38}^{2}$ & 216.9786 & 8403072.8621 & $\widehat{\mathrm{S}}_{\mathrm{p}_{38}}^{2}$ & 21.2121 & 7524802.2329 \\
\hline$\widehat{\mathrm{S}}_{39}^{2}$ & 235.9633 & 8500271.5781 & $\widehat{\mathrm{S}}_{\mathrm{p}_{39}}^{2}$ & 30.4083 & 7588699.7565 \\
\hline$\widehat{S}_{40}^{2}$ & 255.1960 & 8598934.7234 & $\widehat{\mathrm{S}}_{\mathrm{p}_{40}}^{2}$ & 46.8929 & 7698842.0318 \\
\hline$\widehat{S}_{41}^{2}$ & 233.9686 & 8490049.7272 & $\widehat{\mathrm{S}}_{\mathrm{p}_{41}}^{2}$ & 29.2015 & 7580442.3507 \\
\hline$\widehat{\mathrm{S}}_{42}^{2}$ & 261.8741 & 8633234.9291 & $\widehat{\mathrm{S}}_{\mathrm{p}_{42}}^{2}$ & 55.7610 & 7756593.1089 \\
\hline$\widehat{\mathrm{S}}_{43}^{2}$ & 256.6540 & 8606421.4282 & $\widehat{\mathrm{S}}_{\mathrm{p}_{43}}^{2}$ & 48.6422 & 7710301.9522 \\
\hline$\widehat{\mathrm{S}}_{44}^{2}$ & 252.1872 & 8583487.6588 & $\widehat{\mathrm{S}}_{\mathrm{p}_{44}}^{2}$ & 43.5628 & 7676923.4831 \\
\hline$\widehat{\mathrm{S}}_{45}^{2}$ & 250.2947 & 8573774.1884 & $\widehat{\mathrm{S}}_{\mathrm{p}_{45}}^{2}$ & 41.6428 & 7664219.6974 \\
\hline$\widehat{S}_{46}^{2}$ & 243.5012 & 8538919.0760 & $\widehat{\mathrm{S}}_{\mathrm{p}_{46}}^{2}$ & 35.6790 & 7624408.3942 \\
\hline$\widehat{\mathrm{S}}_{47}^{2}$ & 229.6949 & 8468156.2743 & $\widehat{S}_{\mathrm{p}_{47}}^{2}$ & 26.8362 & 7564156.3652 \\
\hline$\widehat{\mathrm{S}}_{48}^{2}$ & 219.4277 & 8415600.5132 & $\widehat{\mathrm{S}}_{\mathrm{p}_{48}}^{2}$ & 22.1574 & 7531487.3776 \\
\hline$\widehat{S}_{49}^{2}$ & 212.3024 & 8379163.5680 & $\widehat{\mathrm{S}}_{\mathrm{p}_{49}}^{2}$ & 19.5567 & 7513015.7817 \\
\hline$\widehat{\mathrm{S}}_{50}^{2}$ & 200.7812 & 8320314.8836 & $\widehat{S}_{\mathrm{p}_{50}}^{2}$ & 16.1672 & 7488520.5228 \\
\hline$\widehat{\mathrm{S}}_{51}^{2}$ & 164.4345 & 8135286.8030 & $\widehat{\mathrm{S}}_{\mathrm{p}_{51}}^{2}$ & 9.4709 & 7438144.1980 \\
\hline$\widehat{S}_{52}^{2}$ & 87.3340 & 7957053.4638 & & & \\
\hline$\widehat{\mathrm{S}}_{\mathrm{R}}^{2}$ & 210.6359 & 8709947.6355 & & & \\
\hline
\end{tabular}

From the values of table 2 and table 3, it is observed that the bias of the proposed modified ratio type variance estimators are less than the bias of the traditional and existing modified ratio type variance estimators. Similarly, it is observed that the mean squared error of the proposed modified ratio type variance estimators are less than the mean squared error of the traditional and existing modified ratio type variance estimators.

\section{CONCLUSION}

In this paper a class of modified ratio type variance estimators has been proposed using the known parameters of the auxiliary variable. The bias and mean squared error of the proposed modified ratio type variance estimators are derived. Further we have derived the conditions for which the proposed estimators are more efficient than the traditional and existing modified ratio type variance estimators. We have also assessed the performances of the proposed estimators with that of the existing estimators for two natural populations. It is observed from the numerical comparison 
that the bias and mean squared error of the proposed estimators are less than the bias and mean squared error of the traditional and existing estimators. Hence we strongly recommend that the proposed modified ratio type variance estimators may be preferred over the traditional ratio type variance estimator and modified ratio type variance estimators for the use of practical applications.

\section{ACKNOWLEDGMENT}

The first author wishes to record his gratitude and thanks to UGC-MRP, New Delhi, for the financial assistance.

\section{REFERENCES}

[1] AGARWAL, M.C. and SITHAPIT, A.B. 1995. Unbiased ratio type estimation. Statistics and Probability Letters, 25: 361-364

[2] AHMED, M.S., RAMAN, M.S. and HOSSAIN, M.I. 2000. Some competitive estimators of finite population variance multivariate auxiliary information. Information and Management Sciences, 11 (1): 49-54

[3] AL-JARARHA, J. and AL-HAJ EBRAHEM, M. 2012. A ratio estimator under general sampling design. Austrian Journal of Statistics, 41(2): 105-115

[4] ARCOS, A., RUEDA, M., MARTINEZ, M.D., GONZALEZ, S. and ROMAN, Y. 2005. Incorporating the auxiliary information available in variance estimation. Applied Mathematics and Computation, 160: 387-399

[5] COCHRAN, W. G. 1977: Sampling techniques, Third Edition, Wiley Eastern Limited

[6] DAS, A.K. and TRIPATHI, T.P. 1978. Use of auxiliary information in estimating the finite population variance. Sankhya, 40: 139-148

[7] GARCIA, M.K. and CEBRAIN, A.A. 1997. Variance estimation using auxiliary information: An almost unbiased multivariate ratio estimator. Metrika, 45: 171-178

[8] GUPTA, S. and SHABBIR, J. 2008. Variance estimation in simple random sampling using auxiliary information. Hacettepe Journal of Mathematics and Statistics, 37: 57-67

[9] ISAKI, C.T. 1983. Variance estimation using auxiliary information. Journal of the American Statistical Association, 78: 117-123

[10] KADILAR, C. and CINGI, H. 2006a. Improvement in variance estimation using auxiliary information. Hacettepe Journal of Mathematics and Statistics, 35 (1): 111-115

[11] KADILAR, C. and CINGI, H. 2006b. Ratio estimators for population variance in simple and stratified sampling. Applied Mathematics and Computation, 173: 1047-1058

[12] MURTHY, M.N. 1967. Sampling theory and methods. Statistical Publishing Society, Calcutta, India

[13] PRASAD, B. and SINGH, H.P. 1990. Some improved ratio type estimators of finite population variance in sample surveys. Communication in Statistics: Theory and Methods, 19: 1127-1139

[14] REDDY, V.N. 1974. On a transformed ratio method of estimation, Sankhya C, 36: 59-70

[15] SHABBIR, J. and GUPTA, S. 2006. On estimation of finite population variance. Journal of Interdisciplinary Mathematics, 9(2), 405-419

[16] SINGH, D. and CHAUDHARY, F.S. 1986. Theory and analysis of sample survey designs. New Age International Publisher 
[17] SINGH, H.P. and SOLANKI, R.S. 2013. A new procedure for variance estimation in simple random sampling using auxiliary information. Statistical Papers, 54, 479-497

[18] SINGH, H.P., UPADHYAYA, U.D. and NAMJOSHI, U.D. 1988. Estimation of finite population variance. Current Science, 57: 1331-1334

[19] SISODIA, B.V.S. and DWIVEDI, V.K. 1981. A modified ratio estimator using coefficient of variation of auxiliary variable. Journal of the Indian Society of Agricultural Statistics, 33(1): 13-18

[20] SUBRAMANI, J. and KUMARAPANDIYAN, G. 2012a. Variance estimation using median of the auxiliary variable. International Journal of Probability and Statistics, Vol. 1(3), 36-40

[21] SUBRAMANI, J. and KUMARAPANDIYAN, G. 2012b. Variance estimation using quartiles and their functions of an auxiliary variable, International Journal of Statistics and Applications, 2012, Vol. 2(5), 67-42

[22] SUBRAMANI, J. and KUMARAPANDIYAN, G. 2012c. Estimation of variance using deciles of an auxiliary variable. Proceedings of International Conference on Frontiers of Statistics and Its Applications, Bonfring Publisher, 143-149

[23] SUBRAMANI, J. and KUMARAPANDIYAN, G. 2013. Estimation of variance using known coefficient of variation and median of an auxiliary variable. Journal of Modern Applied Statistical Methods, Vol. 12(1), 58-64

[24] TAILOR, R. and SHARMA, B. 2012. Modified estimators of population variance in presence of auxiliary information. Statistics in Transition-New series, 13(1), 37-46

[25] UPADHYAYA, L. N. and SINGH, H.P. 2006. Almost unbiased ratio and product-type estimators of finite population variance in sample surveys. Statistics in Transition, 7 (5): 1087-1096

[26] UPADHYAYA, L.N. and SINGH, H.P. 1999. An estimator for population variance that utilizes the kurtosis of an auxiliary variable in sample surveys. Vikram Mathematical Journal, 19, 14-17

[27] UPADHYAYA, L.N. and SINGH, H.P. 2001. Estimation of population standard deviation using auxiliary information. American Journal of Mathematics and Management Sciences, 21(3-4), 345358

[28] WOLTER, K.M. 1985. Introduction to Variance Estimation. Springer-Verlag

[29] YADAV, S.K. and KADILAR, C. 2013a. A class of ratio-cum-dual to ratio estimator of population variance. Journal of Reliability and Statistical Studies, 6(1), 29-34

[30] YADAV, S.K. and KADILAR, C. 2013b. Improved Exponential type ratio estimator of population variance. Colombian Journal of Statistics, 36(1), 145-152 


\section{Appendix-A}

We have derived the expression for the bias and mean squared error of the proposed estimators $\widehat{\mathrm{S}}_{\mathrm{p}_{\mathrm{i}}}^{2}: \mathrm{i}=$ $1,2,3, \ldots, 51$ to first order of approximation with the following notations:

Let $\mathrm{e}_{0}=\frac{\mathrm{s}_{\mathrm{y}}^{2}-\mathrm{S}_{\mathrm{y}}^{2}}{\mathrm{~S}_{\mathrm{y}}^{2}}$ and $\mathrm{e}_{1}=\frac{\overline{\mathrm{x}}-\overline{\mathrm{X}}}{\overline{\mathrm{X}}}$. Further we can write $\mathrm{s}_{\mathrm{y}}^{2}=\mathrm{S}_{\mathrm{y}}^{2}\left(1+\mathrm{e}_{0}\right)$

and $\overline{\mathrm{x}}=\overline{\mathrm{X}}\left(1+\mathrm{e}_{1}\right)$

and from the definition of $\mathrm{e}_{0}$ and $\mathrm{e}_{1}$ we obtain:

$\mathrm{E}\left[\mathrm{e}_{0}\right]=\mathrm{E}\left[\mathrm{e}_{1}\right]=0$

$\mathrm{E}\left[\mathrm{e}_{0}^{2}\right]=\frac{(1-\mathrm{f})}{\mathrm{n}}\left(\beta_{2(\mathrm{y})}-1\right)$

$E\left[e_{1}^{2}\right]=\frac{(1-f)}{n} C_{x}^{2}$

$\mathrm{E}\left[\mathrm{e}_{0} \mathrm{e}_{1}\right]=\frac{(1-\mathrm{f})}{\mathrm{n}} \lambda_{21} \mathrm{C}_{\mathrm{x}}$

The bias of the proposed estimators $\widehat{S}_{\mathrm{p}_{\mathrm{i}}}^{2}: \mathrm{i}=1,2,3, \ldots, 51$ is derived as given below:

$$
\begin{aligned}
& \widehat{S}_{\mathrm{p}_{\mathrm{i}}}^{2}=\mathrm{s}_{\mathrm{y}}^{2}\left[\frac{\left.\overline{\mathrm{X}}+\eta_{\mathrm{i}}\right] ; i=1,2,3, \ldots, 51}{\overline{\mathrm{X}}+\eta_{\mathrm{i}}}\right] ; \hat{\mathrm{S}}_{\mathrm{p}_{\mathrm{i}}}^{2}=\frac{\mathrm{s}_{\mathrm{y}}^{2}}{\left(\overline{\mathrm{X}}+\mathrm{e}_{1} \overline{\mathrm{X}}+\eta_{\mathrm{i}}\right)}\left(\overline{\mathrm{X}}+\eta_{\mathrm{i}}\right) \\
& \Rightarrow \widehat{S}_{\mathrm{p}_{\mathrm{i}}}^{2}=\frac{\mathrm{s}_{\mathrm{y}}^{2}}{\left(\overline{\mathrm{X}}+\eta_{\mathrm{i}}\right)\left(1+\frac{\mathrm{e}_{1} \overline{\mathrm{X}}}{\bar{X}+\eta_{\mathrm{i}}}\right)}\left(\overline{\mathrm{X}}+\eta_{\mathrm{i}}\right) \\
& \Rightarrow \widehat{S}_{\mathrm{p}_{\mathrm{i}}}^{2}=\frac{\mathrm{s}_{\mathrm{y}}^{2}}{\left(1+\theta_{\mathrm{p}_{\mathrm{i}}} \mathrm{e}_{1}\right)} \mathrm{where} \theta_{\mathrm{p}_{\mathrm{i}}}=\frac{\overline{\mathrm{X}}}{\overline{\mathrm{X}}+\eta_{\mathrm{i}}} \\
& \Rightarrow \widehat{S}_{\mathrm{p}_{\mathrm{i}}}^{2}=\mathrm{s}_{\mathrm{y}}^{2}\left(1+\theta_{\mathrm{p}_{\mathrm{i}}} \mathrm{e}_{1}\right)^{-1} \\
& \Rightarrow \widehat{S}_{\mathrm{p}_{\mathrm{i}}}^{2}=\mathrm{s}_{\mathrm{y}}^{2}\left(1-\theta_{\mathrm{p}_{\mathrm{i}}} \mathrm{e}_{1}+\theta_{\mathrm{p}_{\mathrm{i}}}^{2} \mathrm{e}_{1}^{2}-\theta_{\mathrm{p}_{\mathrm{i}}}^{3} \mathrm{e}_{1}^{3}+\cdots\right)
\end{aligned}
$$

Neglecting the terms more than $2^{\text {nd }}$ order, we will get

$$
\begin{aligned}
& \widehat{S}_{\mathrm{p}_{\mathrm{i}}}^{2}=\mathrm{s}_{\mathrm{y}}^{2}\left(1-\theta_{\mathrm{p}_{\mathrm{i}}} \mathrm{e}_{1}+\theta_{\mathrm{p}_{\mathrm{i}}}^{2} \mathrm{e}_{1}^{2}\right) \\
& \Rightarrow \widehat{\mathrm{S}}_{\mathrm{p}_{\mathrm{i}}}^{2}=\left(\mathrm{S}_{\mathrm{y}}^{2}\left(1+\mathrm{e}_{0}\right)\right)\left(1-\theta_{\mathrm{p}_{\mathrm{i}}} \mathrm{e}_{1}+\theta_{\mathrm{p}_{\mathrm{i}}}^{2} \mathrm{e}_{1}^{2}\right) \\
& \Rightarrow \widehat{\mathrm{S}}_{\mathrm{p}_{\mathrm{i}}}^{2}=\left(\mathrm{S}_{\mathrm{y}}^{2}+\mathrm{S}_{\mathrm{y}}^{2} \mathrm{e}_{0}\right)\left(1-\theta_{\mathrm{p}_{\mathrm{i}}} \mathrm{e}_{1}+\theta_{\mathrm{p}_{\mathrm{i}}}^{2} \mathrm{e}_{1}^{2}\right) \\
& \Rightarrow \widehat{\mathrm{S}}_{\mathrm{p}_{\mathrm{i}}}^{2}=\mathrm{S}_{\mathrm{y}}^{2}+\mathrm{S}_{\mathrm{y}}^{2} \mathrm{e}_{0}-\mathrm{S}_{\mathrm{y}}^{2} \theta_{\mathrm{p}_{\mathrm{i}}} \mathrm{e}_{1}-\mathrm{S}_{\mathrm{y}}^{2} \theta_{\mathrm{p}_{\mathrm{i}}} \mathrm{e}_{0} \mathrm{e}_{1}+\mathrm{S}_{\mathrm{y}}^{2} \theta_{\mathrm{p}_{\mathrm{i}}}^{2} \mathrm{e}_{1}^{2}+\mathrm{S}_{\mathrm{y}}^{2} \theta_{\mathrm{p}_{\mathrm{i}}}^{2} \mathrm{e}_{0} \mathrm{e}_{1}^{2}
\end{aligned}
$$

Neglecting the terms more than $3^{\text {rd }}$ order, we will get 


$$
\begin{aligned}
& \widehat{S}_{\mathrm{p}_{\mathrm{i}}}^{2}=\mathrm{S}_{\mathrm{y}}^{2}+\mathrm{S}_{\mathrm{y}}^{2} \mathrm{e}_{0}-S_{y}^{2} \theta_{\mathrm{p}_{\mathrm{i}}} \mathrm{e}_{1}-S_{\mathrm{y}}^{2} \theta_{\mathrm{p}_{\mathrm{i}}} \mathrm{e}_{0} \mathrm{e}_{1}+S_{y}^{2} \theta_{\mathrm{p}_{\mathrm{i}}}^{2} \mathrm{e}_{1}^{2} \\
& \Rightarrow \widehat{S}_{\mathrm{p}_{\mathrm{i}}}^{2}-S_{\mathrm{y}}^{2}=S_{\mathrm{y}}^{2} \mathrm{e}_{0}-S_{\mathrm{y}}^{2} \theta_{\mathrm{p}_{\mathrm{i}}} \mathrm{e}_{1}-S_{\mathrm{y}}^{2} \theta_{\mathrm{p}_{\mathrm{i}}} \mathrm{e}_{0} \mathrm{e}_{1}+\mathrm{S}_{\mathrm{y}}^{2} \theta_{\mathrm{p}_{\mathrm{i}}}^{2} \mathrm{e}_{1}^{2}
\end{aligned}
$$

Taking expectation on both sides, we will get

$$
\begin{aligned}
& E\left(\hat{S}_{p_{i}}^{2}-S_{y}^{2}\right)=S_{y}^{2} E\left(e_{0}\right)-S_{y}^{2} \theta_{p_{i}} E\left(e_{1}\right)-S_{y}^{2} \theta_{p_{i}} E\left(e_{0} e_{1}\right)+S_{y}^{2} \theta_{p_{i}}^{2} E\left(e_{1}^{2}\right) \\
& \Rightarrow \operatorname{Bias}\left(\hat{S}_{p_{i}}^{2}\right)=S_{y}^{2} \theta_{p_{i}}^{2} E\left(e_{1}^{2}\right)-S_{y}^{2} \theta_{p_{i}} E\left(e_{0} e_{1}\right) \\
& \Rightarrow \operatorname{Bias}\left(\widehat{S}_{p_{i}}^{2}\right)=S_{y}^{2} \theta_{p_{i}}^{2} \frac{(1-f)}{n} C_{x}^{2}-S_{y}^{2} \frac{(1-f)}{n} \lambda_{21} C_{x} \theta_{p_{i}} \\
& \Rightarrow \operatorname{Bias}\left(\widehat{S}_{p_{i}}^{2}\right)=\frac{(1-f)}{n}\left(S_{y}^{2} \theta_{p_{i}}^{2} C_{x}^{2}-S_{y}^{2} \lambda_{21} C_{x} \theta_{p_{i}}\right) \\
& \Rightarrow \operatorname{Bias}\left(\widehat{S}_{p_{i}}^{2}\right)=\gamma S_{y}^{2}\left(\theta_{p_{i}}^{2} C_{x}^{2}-\theta_{p_{i}} \lambda_{21} C_{x}\right) \text { where } \theta_{p_{i}}=\frac{\bar{X}}{\bar{X}+\eta_{i}}
\end{aligned}
$$

The mean squared error of the class of proposed estimators $\widehat{S}_{\mathrm{p}_{\mathrm{i}}}^{2}: \mathrm{i}=1,2,3, \ldots, 51$ to first order of approximation is derived as given below:

$$
\begin{aligned}
& \hat{S}_{\mathrm{p}_{\mathrm{i}}}^{2}=\mathrm{s}_{\mathrm{y}}^{2}\left[\frac{\overline{\mathrm{X}}+\eta_{\mathrm{i}}}{\overline{\mathrm{x}}+\eta_{\mathrm{i}}}\right] ; \mathrm{i}=1,2,3, \ldots, 51 \\
& \Rightarrow \widehat{S}_{\mathrm{p}_{\mathrm{i}}}^{2}=\frac{\mathrm{s}_{\mathrm{y}}^{2}}{\left(\overline{\mathrm{X}}+\mathrm{e}_{1} \overline{\mathrm{X}}+\eta_{\mathrm{i}}\right)}\left(\overline{\mathrm{X}}+\eta_{\mathrm{i}}\right) \\
& \Rightarrow \hat{\mathrm{S}}_{\mathrm{p}_{\mathrm{i}}}^{2}=\frac{\mathrm{s}_{\mathrm{y}}^{2}}{\left(\overline{\mathrm{X}}+\eta_{\mathrm{i}}\right)\left(1+\frac{\mathrm{e}_{1} \overline{\mathrm{X}}}{\overline{\mathrm{X}}+\eta_{\mathrm{i}}}\right)}\left(\overline{\mathrm{X}}+\eta_{\mathrm{i}}\right) \\
& \Rightarrow \hat{\mathrm{S}}_{\mathrm{p}_{\mathrm{i}}}^{2}=\frac{\mathrm{s}_{\mathrm{y}}^{2}}{\left(1+\theta_{\mathrm{p}_{\mathrm{i}}} \mathrm{e}_{1}\right)} \text { where } \theta_{\mathrm{p}_{\mathrm{i}}}=\frac{\overline{\mathrm{X}}}{\overline{\mathrm{X}}+\eta_{\mathrm{i}}} \\
& \Rightarrow \hat{S}_{\mathrm{p}_{\mathrm{i}}}^{2}=\mathrm{s}_{\mathrm{y}}^{2}\left(1+\theta_{\mathrm{p}_{\mathrm{i}}} \mathrm{e}_{1}\right)^{-1} \\
& \Rightarrow \widehat{S}_{\mathrm{p}_{\mathrm{i}}}^{2}=\mathrm{s}_{\mathrm{y}}^{2}\left(1-\theta_{\mathrm{p}_{\mathrm{i}}} \mathrm{e}_{1}+\theta_{\mathrm{p}_{\mathrm{i}}}^{2} \mathrm{e}_{1}^{2}-\theta_{\mathrm{p}_{\mathrm{i}}}^{3} \mathrm{e}_{1}^{3}+\cdots\right)
\end{aligned}
$$

Neglecting the terms more than $1^{\text {st }}$ order, we will get

$$
\begin{aligned}
& \Rightarrow \hat{S}_{\mathrm{p}_{\mathrm{i}}}^{2}=\mathrm{s}_{\mathrm{y}}^{2}\left(1-\theta_{\mathrm{p}_{\mathrm{i}}} \mathrm{e}_{1}\right) \\
& \Rightarrow \widehat{S}_{\mathrm{p}_{\mathrm{i}}}^{2}=\left(\mathrm{S}_{\mathrm{y}}^{2}\left(1+\mathrm{e}_{0}\right)\right)\left(1-\theta_{\mathrm{p}_{\mathrm{i}}} \mathrm{e}_{1}\right) \\
& \Rightarrow \hat{\mathrm{S}}_{\mathrm{p}_{\mathrm{i}}}^{2}=\left(\mathrm{S}_{\mathrm{y}}^{2}+\mathrm{S}_{\mathrm{y}}^{2} \mathrm{e}_{0}\right)\left(1-\theta_{\mathrm{p}_{\mathrm{i}}} \mathrm{e}_{1}\right) \\
& \Rightarrow \hat{S}_{\mathrm{p}_{\mathrm{i}}}^{2}=\mathrm{S}_{\mathrm{y}}^{2}+\mathrm{S}_{\mathrm{y}}^{2} \mathrm{e}_{0}-\mathrm{S}_{\mathrm{y}}^{2} \theta_{\mathrm{p}_{\mathrm{i}}} \mathrm{e}_{1}-S_{\mathrm{y}}^{2} \theta_{\mathrm{p}_{\mathrm{i}}} \mathrm{e}_{0} \mathrm{e}_{1} \\
& \Rightarrow \hat{S}_{\mathrm{p}_{\mathrm{i}}}^{2}-S_{\mathrm{y}}^{2}=S_{y}^{2} \mathrm{e}_{0}-S_{\mathrm{y}}^{2} \theta_{\mathrm{p}_{\mathrm{i}}} \mathrm{e}_{1}-S_{y}^{2} \theta_{\mathrm{p}_{\mathrm{i}}} \mathrm{e}_{0} \mathrm{e}_{1}
\end{aligned}
$$


Squaring both sides

$\Rightarrow\left(\widehat{\mathrm{S}}_{\mathrm{p}_{\mathrm{i}}}^{2}-\mathrm{S}_{\mathrm{y}}^{2}\right)^{2}=\left(\mathrm{S}_{\mathrm{y}}^{2} \mathrm{e}_{0}-\mathrm{S}_{\mathrm{y}}^{2} \theta_{\mathrm{p}_{\mathrm{i}}} \mathrm{e}_{1}-\mathrm{S}_{\mathrm{y}}^{2} \theta_{\mathrm{p}_{\mathrm{i}}} \mathrm{e}_{0} \mathrm{e}_{1}\right)^{2}$

Neglecting the terms more than $2^{\text {nd }}$ order, we will get

$\left(\hat{S}_{\mathrm{p}_{\mathrm{i}}}^{2}-\mathrm{S}_{\mathrm{y}}^{2}\right)^{2}=\mathrm{S}_{\mathrm{y}}^{4} \mathrm{e}_{0}{ }^{2}+\mathrm{S}_{\mathrm{y}}^{4} \theta_{\mathrm{p}_{\mathrm{i}}}^{2} \mathrm{e}_{1}^{2}-2 \mathrm{~S}_{\mathrm{y}}^{4} \theta_{\mathrm{p}_{\mathrm{i}}} \mathrm{e}_{0} \mathrm{e}_{1}$

Taking expectation on both sides we will get:

$$
\begin{aligned}
& \mathrm{E}\left(\hat{\mathrm{S}}_{\mathrm{p}_{\mathrm{i}}}^{2}-\mathrm{S}_{\mathrm{y}}^{2}\right)^{2}=S_{\mathrm{y}}^{4} \mathrm{E}\left(\mathrm{e}_{0}^{2}\right)+\mathrm{S}_{\mathrm{y}}^{4} \theta_{\mathrm{p}_{\mathrm{i}}}^{2} \mathrm{E}\left(\mathrm{e}_{1}^{2}\right)-2 S_{\mathrm{y}}^{4} \theta_{\mathrm{p}_{\mathrm{i}}} \mathrm{E}\left(\mathrm{e}_{0} \mathrm{e}_{1}\right) \\
& \Rightarrow \operatorname{MSE}\left(\hat{\mathrm{S}}_{\mathrm{p}_{\mathrm{i}}}^{2}\right)=\frac{(1-\mathrm{f})}{\mathrm{n}}\left(\mathrm{S}_{\mathrm{y}}^{4}\left(\beta_{2(\mathrm{y})}-1\right)+\mathrm{S}_{\mathrm{y}}^{4} \theta_{\mathrm{p}_{\mathrm{i}}}^{2} \mathrm{C}_{\mathrm{x}}^{2}-2 \mathrm{~S}_{\mathrm{y}}^{4} \theta_{\mathrm{p}_{\mathrm{i}}} \lambda_{21} \mathrm{C}_{\mathrm{x}}\right) \\
& \Rightarrow \operatorname{MSE}\left(\hat{\mathrm{S}}_{\mathrm{p}_{\mathrm{i}}}^{2}\right)=\frac{(1-\mathrm{f})}{\mathrm{n}} \mathrm{S}_{\mathrm{y}}^{4}\left(\left(\beta_{2(\mathrm{y})}-1\right)+\theta_{\mathrm{p}_{\mathrm{i}}}^{2} \mathrm{C}_{\mathrm{x}}^{2}-2 \theta_{\mathrm{p}_{\mathrm{i}}} \lambda_{21} \mathrm{C}_{\mathrm{x}}\right) ; \mathrm{i}=1,2,3, \ldots, 51 \\
& \Rightarrow \operatorname{MSE}\left(\hat{\mathrm{S}}_{\mathrm{p}_{\mathrm{i}}}^{2}\right)=\gamma \mathrm{S}_{\mathrm{y}}^{4}\left(\left(\beta_{2(\mathrm{y})}-1\right)+\theta_{\mathrm{p}_{\mathrm{i}}}^{2} \mathrm{C}_{\mathrm{x}}^{2}-2 \theta_{\mathrm{p}_{\mathrm{i}}} \lambda_{21} \mathrm{C}_{\mathrm{x}}\right) ; \mathrm{i}=1,2,3, \ldots, 51
\end{aligned}
$$

\section{Appendix-B}

The conditions for which proposed estimators $\widehat{\mathrm{S}}_{\mathrm{p}_{\mathrm{i}}}^{2}$ perform better than the traditional ratio type variance estimator $\widehat{\mathrm{S}}_{\mathrm{R}}^{2}$ are derived and are given below:

\section{$\operatorname{For} \operatorname{MSE}\left(\hat{\mathbf{S}}_{\mathbf{p}_{\mathbf{i}}}^{2}\right) \leq \operatorname{MSE}\left(\widehat{\mathbf{S}}_{\mathbf{R}}^{2}\right)$}

$$
\begin{aligned}
& \gamma S_{\mathrm{y}}^{4}\left[\left(\beta_{2(\mathrm{y})}-1\right)+\theta_{\mathrm{p}_{\mathrm{i}}}^{2} \mathrm{C}_{\mathrm{x}}^{2}-2 \theta_{\mathrm{p}_{\mathrm{i}}} \lambda_{21} \mathrm{C}_{\mathrm{x}}\right] \leq \gamma \mathrm{S}_{\mathrm{y}}^{4}\left[\left(\beta_{2(\mathrm{y})}-1\right)+\left(\beta_{2(\mathrm{x})}-1\right)-2\left(\lambda_{22}-1\right)\right] \\
& \Rightarrow\left(\left(\beta_{2(\mathrm{y})}-1\right)+\theta_{\mathrm{p}_{\mathrm{i}}}^{2} \mathrm{C}_{\mathrm{x}}^{2}-2 \theta_{\mathrm{p}_{\mathrm{i}}} \lambda_{21} \mathrm{C}_{\mathrm{x}}\right) \leq\left[\left(\beta_{2(\mathrm{y})}-1\right)+\left(\beta_{2(\mathrm{x})}-1\right)-2\left(\lambda_{22}-1\right)\right] \\
& \Rightarrow \theta_{\mathrm{p}_{\mathrm{i}}}^{2} \mathrm{C}_{\mathrm{x}}^{2}-2 \theta_{\mathrm{p}_{\mathrm{i}}} \lambda_{21} \mathrm{C}_{\mathrm{x}} \leq\left(\beta_{2(\mathrm{x})}-1\right)-2\left(\lambda_{22}-1\right) \\
& \Rightarrow \theta_{\mathrm{p}_{\mathrm{i}}}^{2} \mathrm{C}_{\mathrm{x}}^{2}-2 \theta_{\mathrm{p}_{\mathrm{i}}} \lambda_{21} \mathrm{C}_{\mathrm{x}}+\lambda_{21}^{2}-\lambda_{21}^{2} \leq\left(\beta_{2(\mathrm{x})}-1\right)-2\left(\lambda_{22}-1\right) \\
& \Rightarrow\left(\theta_{\mathrm{p}_{\mathrm{i}}} \mathrm{C}_{\mathrm{x}}-\lambda_{21}\right)^{2} \leq\left(\beta_{2(\mathrm{x})}-1\right)-2\left(\lambda_{22}-1\right)+\lambda_{21}^{2} \\
& \Rightarrow\left(\theta_{\mathrm{p}_{\mathrm{i}}} \mathrm{C}_{\mathrm{x}}-\lambda_{21}\right) \leq\left(\left(\beta_{2(\mathrm{x})}-1\right)-2\left(\lambda_{22}-1\right)+\lambda_{21}^{2}\right)^{\frac{1}{2}} \\
& \Rightarrow \theta_{\mathrm{p}_{\mathrm{i}}} \mathrm{C}_{\mathrm{x}} \leq \lambda_{21}+\left(\left(\beta_{2(\mathrm{x})}-1\right)-2\left(\lambda_{22}-1\right)+\lambda_{21}^{2}\right)^{\frac{1}{2}} \\
& \Rightarrow \theta_{\mathrm{p}_{\mathrm{i}}} \leq \frac{\lambda_{21}+\left(\left(\beta_{2(\mathrm{x})}-1\right)-2\left(\lambda_{22}-1\right)+\lambda_{21}^{2}\right)^{\frac{1}{2}}}{\mathrm{C}_{\mathrm{x}}}
\end{aligned}
$$

That is, $\operatorname{MSE}\left(\hat{\mathrm{S}}_{\mathrm{p}_{\mathrm{i}}}^{2}\right) \leq \operatorname{MSE}\left(\widehat{\mathrm{S}}_{\mathrm{R}}^{2}\right)$ if $\theta_{\mathrm{p}_{\mathrm{i}}} \leq \frac{\lambda_{21}+\left(\left(\beta_{2(\mathrm{x})}-1\right)-2\left(\lambda_{22}-1\right)+\lambda_{21}^{2}\right)^{\frac{1}{2}}}{\mathrm{C}_{\mathrm{x}}}$ 


\section{Appendix-C}

The conditions for which proposed estimators $\widehat{S}_{\mathrm{p}_{\mathrm{i}}}^{2}$ perform better than the existing modified ratio type variance estimators $\widehat{S}_{\mathrm{i}}^{2}$ are derived and are given below:

$\operatorname{For} \operatorname{MSE}\left(\hat{\mathbf{S}}_{\mathbf{p}_{\mathbf{i}}}^{2}\right) \leq \operatorname{MSE}\left(\hat{\mathbf{S}}_{\mathbf{i}}^{2}\right)$

$$
\begin{aligned}
& \gamma S_{\mathrm{y}}^{4}\left[\left(\beta_{2(\mathrm{y})}-1\right)+\theta_{\mathrm{p}_{\mathrm{i}}}^{2} \mathrm{C}_{\mathrm{x}}^{2}-2 \theta_{\mathrm{p}_{\mathrm{i}}} \lambda_{21} \mathrm{C}_{\mathrm{x}}\right] \leq \gamma S_{\mathrm{y}}^{4}\left[\left(\beta_{2(\mathrm{y})}-1\right)+\delta_{\mathrm{i}}^{2}\left(\beta_{2(\mathrm{x})}-1\right)-2 \delta_{\mathrm{i}}\left(\lambda_{22}-1\right)\right] \\
& \Rightarrow\left(\left(\beta_{2(\mathrm{y})}-1\right)+\theta_{\mathrm{p}_{\mathrm{i}}}^{2} \mathrm{C}_{\mathrm{x}}^{2}-2 \theta_{\mathrm{p}_{\mathrm{i}}} \lambda_{21} \mathrm{C}_{\mathrm{x}}\right) \leq\left[\left(\beta_{2(\mathrm{y})}-1\right)+\delta_{\mathrm{i}}^{2}\left(\beta_{2(\mathrm{x})}-1\right)-2 \delta_{\mathrm{i}}\left(\lambda_{22}-1\right)\right] \\
& \Rightarrow \theta_{\mathrm{p}_{\mathrm{i}}}^{2} C_{\mathrm{x}}^{2}-2 \theta_{\mathrm{p}_{\mathrm{i}}} \lambda_{21} \mathrm{C}_{\mathrm{x}} \leq \delta_{\mathrm{i}}^{2}\left(\beta_{2(\mathrm{x})}-1\right)-2 \delta_{\mathrm{i}}\left(\lambda_{22}-1\right) \\
& \Rightarrow \theta_{\mathrm{p}_{\mathrm{i}}}^{2} C_{\mathrm{x}}^{2}-2 \theta_{\mathrm{p}_{\mathrm{i}}} \lambda_{21} \mathrm{C}_{\mathrm{x}}+\lambda_{21}^{2}-\lambda_{21}^{2} \leq \delta_{\mathrm{i}}^{2}\left(\beta_{2(\mathrm{x})}-1\right)-2 \delta_{\mathrm{i}}\left(\lambda_{22}-1\right) \\
& \Rightarrow\left(\theta_{\mathrm{p}_{\mathrm{i}}} \mathrm{C}_{\mathrm{x}}-\lambda_{21}\right)^{2} \leq \delta_{\mathrm{i}}^{2}\left(\beta_{2(\mathrm{x})}-1\right)-2 \delta_{\mathrm{i}}\left(\lambda_{22}-1\right)+\lambda_{21}^{2} \\
& \Rightarrow\left(\theta_{\mathrm{p}_{\mathrm{i}}} \mathrm{C}_{\mathrm{x}}-\lambda_{21}\right) \leq\left(\delta_{\mathrm{i}}^{2}\left(\beta_{2(\mathrm{x})}-1\right)-2 \delta_{\mathrm{i}}\left(\lambda_{22}-1\right)+\lambda_{21}^{2}\right)^{\frac{1}{2}} \\
& \Rightarrow \theta_{\mathrm{p}_{\mathrm{i}}} \mathrm{C}_{\mathrm{x}} \leq \lambda_{21}+\left(\delta_{\mathrm{i}}^{2}\left(\beta_{2(\mathrm{x})}-1\right)-2 \delta_{\mathrm{i}}\left(\lambda_{22}-1\right)+\lambda_{21}^{2}\right)^{\frac{1}{2}} \\
& \Rightarrow \theta_{\mathrm{p}_{\mathrm{i}}} \leq \frac{\lambda_{21}+\left(\delta_{\mathrm{i}}^{2}\left(\beta_{2(\mathrm{x})}-1\right)-2 \delta_{\mathrm{i}}\left(\lambda_{22}-1\right)+\lambda_{21}^{2}\right)^{\frac{1}{2}}}{\mathrm{C}_{\mathrm{x}}}
\end{aligned}
$$

That is, $\operatorname{MSE}\left(\widehat{\mathrm{S}}_{\mathrm{p}_{\mathrm{i}}}^{2}\right) \leq \operatorname{MSE}\left(\widehat{\mathrm{S}}_{\mathrm{i}}^{2}\right)$ if $\theta_{\mathrm{p}_{\mathrm{i}}} \leq \frac{\lambda_{21}+\left(\delta_{\mathrm{i}}^{2}\left(\beta_{2(\mathrm{x})}-1\right)-2 \delta_{\mathrm{i}}\left(\lambda_{22}-1\right)+\lambda_{21}^{2}\right)^{\frac{1}{2}}}{\mathrm{C}_{\mathrm{x}}}$

\section{Appendix-D}

The conditions for which proposed estimators $\widehat{S}_{\mathrm{p}_{\mathrm{i}}}^{2}$ perform better than the existing modified ratio type variance estimator $\widehat{S}_{52}^{2}$ are derived and are given below:

$\operatorname{For} \operatorname{MSE}\left(\hat{\mathbf{S}}_{\mathbf{p}_{\mathbf{i}}}^{2}\right) \leq \operatorname{MSE}\left(\hat{\mathbf{S}}_{52}^{2}\right)$

$$
\begin{aligned}
& \gamma S_{y}^{4}\left[\left(\beta_{2(y)}-1\right)+\theta_{\mathrm{p}_{\mathrm{i}}}^{2} C_{\mathrm{x}}^{2}-2 \theta_{\mathrm{p}_{\mathrm{i}}} \lambda_{21} \mathrm{C}_{\mathrm{x}}\right] \leq \gamma S_{\mathrm{y}}^{4}\left[\left(\beta_{2(\mathrm{y})}-1\right)+\mathrm{C}_{\mathrm{x}}^{2}-2 \lambda_{21} \mathrm{C}_{\mathrm{x}}\right] \\
& \Rightarrow\left(\left(\beta_{2(\mathrm{y})}-1\right)+\theta_{\mathrm{p}_{\mathrm{i}}}^{2} C_{\mathrm{x}}^{2}-2 \theta_{\mathrm{p}_{\mathrm{i}}} \lambda_{21} \mathrm{C}_{\mathrm{x}}\right) \leq\left[\left(\beta_{2(\mathrm{y})}-1\right)+\mathrm{C}_{\mathrm{x}}^{2}-2 \lambda_{21} \mathrm{C}_{\mathrm{x}}\right] \\
& \Rightarrow \theta_{\mathrm{p}_{\mathrm{i}}}^{2} C_{\mathrm{x}}^{2}-2 \theta_{\mathrm{p}_{\mathrm{i}}} \lambda_{21} \mathrm{C}_{\mathrm{x}} \leq \mathrm{C}_{\mathrm{x}}^{2}-2 \lambda_{21} \mathrm{C}_{\mathrm{x}} \\
& \Rightarrow \theta_{\mathrm{p}_{\mathrm{i}}}^{2} C_{\mathrm{x}}^{2}-2 \theta_{\mathrm{p}_{\mathrm{i}}} \lambda_{21} \mathrm{C}_{\mathrm{x}}-\mathrm{C}_{\mathrm{x}}^{2}+2 \lambda_{21} \mathrm{C}_{\mathrm{x}} \leq 0 \\
& \Rightarrow\left(\theta_{\mathrm{p}_{\mathrm{i}}}^{2}-1\right) \mathrm{C}_{\mathrm{x}}^{2}-2 \lambda_{21} \mathrm{C}_{\mathrm{x}}\left(\theta_{\mathrm{p}_{\mathrm{i}}}-1\right) \leq 0 \\
& \Rightarrow\left(\theta_{\mathrm{p}_{\mathrm{i}}}+1\right)\left(\theta_{\mathrm{p}_{\mathrm{i}}}-1\right) \mathrm{C}_{\mathrm{x}}^{2}-2 \lambda_{21} \mathrm{C}_{\mathrm{x}}\left(\theta_{\mathrm{p}_{\mathrm{i}}}-1\right) \leq 0 \\
& \Rightarrow\left(\theta_{\mathrm{p}_{\mathrm{i}}}-1\right)\left[\left(\theta_{\mathrm{p}_{\mathrm{i}}}+1\right) \mathrm{C}_{\mathrm{x}}^{2}-2 \lambda_{21} \mathrm{C}_{\mathrm{x}}\right] \leq 0
\end{aligned}
$$

Condition 1: $\left(\theta_{p_{i}}-1\right) \leq 0$ and $\left[\left(\theta_{p_{i}}+1\right) C_{x}^{2}-2 \lambda_{21} C_{x}\right] \geq 0$

$$
\Rightarrow \theta_{\mathrm{p}_{\mathrm{i}}} \leq 1 \text { and }\left(\theta_{\mathrm{p}_{\mathrm{i}}}+1\right) \mathrm{C}_{\mathrm{x}}^{2} \geq 2 \lambda_{21} \mathrm{C}_{\mathrm{x}}
$$




$$
\begin{aligned}
& \Rightarrow \theta_{\mathrm{p}_{\mathrm{i}}} \leq 1 \text { and } \theta_{\mathrm{p}_{\mathrm{i}}} \geq 2 \frac{\lambda_{21}}{\mathrm{C}_{\mathrm{x}}}-1 \\
& \Rightarrow 2 \frac{\lambda_{21}}{\mathrm{C}_{\mathrm{x}}}-1 \leq \theta_{\mathrm{p}_{\mathrm{i}}} \leq 1
\end{aligned}
$$

Condition 2: $\left(\theta_{p_{i}}-1\right) \geq 0$ and $\left[\left(\theta_{p_{i}}+1\right) C_{x}^{2}-2 \lambda_{21} C_{x}\right] \leq 0$

$$
\begin{aligned}
& \Rightarrow \theta_{\mathrm{p}_{\mathrm{i}}} \geq 1 \text { and }\left(\theta_{\mathrm{p}_{\mathrm{i}}}+1\right) \mathrm{C}_{\mathrm{x}}^{2} \leq 2 \lambda_{21} \mathrm{C}_{\mathrm{x}} \\
& \Rightarrow \theta_{\mathrm{p}_{\mathrm{i}}} \geq 1 \text { and } \theta_{\mathrm{p}_{\mathrm{i}}} \leq 2 \frac{\lambda_{21}}{\mathrm{C}_{\mathrm{x}}}-1 \\
& \Rightarrow 1 \leq \theta_{\mathrm{p}_{\mathrm{i}}} \leq 2 \frac{\lambda_{21}}{\mathrm{C}_{\mathrm{x}}}-1
\end{aligned}
$$

That is, $\operatorname{MSE}\left(\widehat{\mathrm{S}}_{\mathrm{p}_{\mathrm{i}}}^{2}\right) \leq \operatorname{MSE}\left(\widehat{\mathrm{S}}_{52}^{2}\right)$ either $2 \frac{\lambda_{21}}{\mathrm{C}_{\mathrm{x}}}-1 \leq \theta_{\mathrm{p}_{\mathrm{i}}} \leq 1$ (or) $1 \leq \theta_{\mathrm{p}_{\mathrm{i}}} \leq 2 \frac{\lambda_{21}}{\mathrm{C}_{\mathrm{x}}}-1$

J. Subramani

Department of Statistics

Ramanujan School of Mathematical Sciences, Pondicherry University

R V Nagar, Kalapet, Puducherry - 605014, India.

Email: drjsubramani@yahoo.co.in

G. Kumarapandiyan

Department of Statistics

Ramanujan School of Mathematical Sciences, Pondicherry University

R V Nagar, Kalapet, Puducherry - 605014, India.

Email: kumarstat88@gmail.com 